Supplement of Hydrol. Earth Syst. Sci., 20, 4775-4799, 2016

http://www.hydrol-earth-syst-sci.net/20/4775/2016/

doi:10.5194/hess-20-4775-2016-supplement

(C) Author(s) 2016. CC Attribution 3.0 License.

(c) (1)

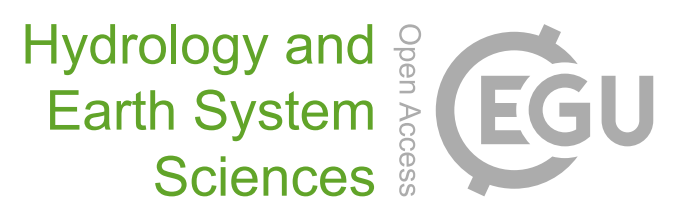

Supplement of

\title{
The evolution of root-zone moisture capacities after deforestation: a step towards hydrological predictions under change?
}

\section{Remko Nijzink et al.}

Correspondence to: Remko Nijzink (r.c.nijzink@tudelft.nl)

The copyright of individual parts of the supplement might differ from the CC-BY 3.0 licence. 


\section{Supplementary Material}

\section{S1. Protocol}

Experiment: The evolution of root zone moisture capacities after land use change

Partners: SMHI, Bristol

Lead: TUD

\section{Formal protocol}

\section{Execution}

i. Data requirements and formatting

Required data: Long-term hydrological data (i.e. daily precipitation, runoff and potential evaporation) for at least 20 years for at least one and temporally well documented, land use change (ideally deforestation). Selected catchments are Hubbard Brook WS2 and WS5, and HJ Andrews WS1.

ii. Experiment execution steps

a. TUD: Determine time series of root zone moisture capacity based on waterbalance.

b. SMHI, Bristol, TUD: Randomly run models with a moving time window of 2 years. Compute Kling-Gupta Efficiency, log Kling-Gupta Efficiency and Volume Error for each model run.

c. TUD: Adjust model with a more dynamic formulation of the root zone storage capacity. Randomly run adjusted model and original model for a longer time series including deforestation. Compute hydrological signatures.

iii. Result reporting

a. Mat-files with the used parameter combinations and performance metrics for each window.

Analysis

i. Trend analysis for the water balance derived root zone storage capacities

ii. Derive posterior distributions for window-based model runs for the root zone storage capacity or the equivalent parameter for each model. 
iii. Calculate probability of improvement and ranked probability score for each hydrological signature for the adjusted model with time-varying root zone storage capacity and the original model.

iv. Analyse/interpret findings. 


\section{S2. Model descriptions}

\subsection{FLEX}

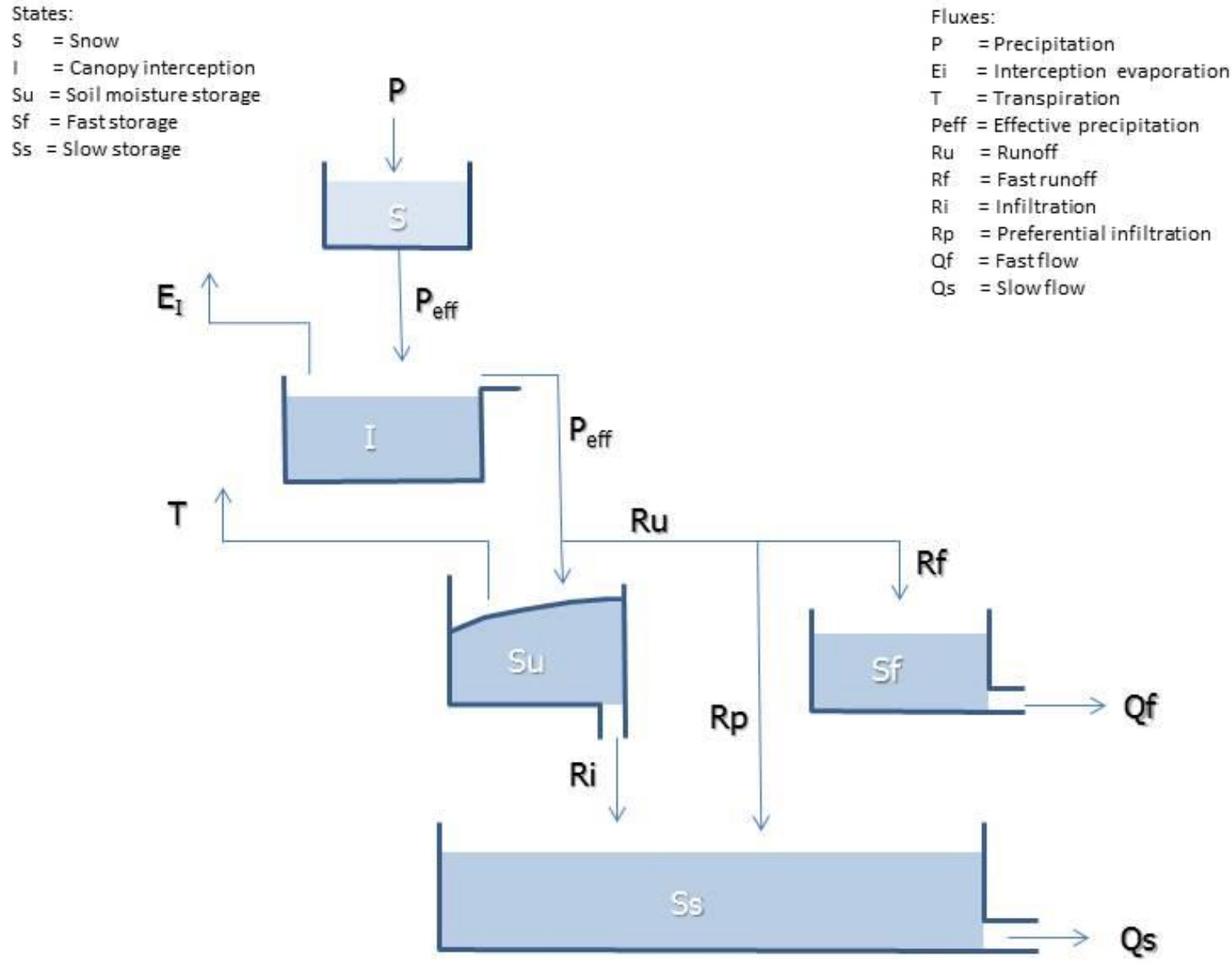

Figure S1. FLEX model structure. 
Table S1. Parameters with descriptions and prior ranges for the FLEX model

\begin{tabular}{|c|c|c|c|c|}
\hline Parameters & Unit & Min & Max & Description \\
\hline Meltfactor & $\mathrm{mm} /{ }^{\circ} \mathrm{C}$ & 4 & 5 & Water released with a degree change temperature \\
\hline Tthresh & ${ }^{\circ} \mathrm{C}$ & -1 & 0 & Threshold temperature to separate rain from snow \\
\hline Imax & $\mathrm{mm}$ & 0.0 & 5 & Maximum interception capacity \\
\hline Sumax & $\mathrm{mm}$ & 1.0 & 1000 & Maximum soil moisture storage \\
\hline Beta & - & 0.01 & 3 & Shape factor soil moisture function \\
\hline Kf & days & 1 & 10 & Recession coefficient fast reservoir \\
\hline Ks & days & 60 & 65 & Recession coefficient slow reservoir \\
\hline D & - & 0.0 & 1.0 & $\begin{array}{l}\text { Partition of runoff that preferentially percolates to } \\
\text { the groundwater }\end{array}$ \\
\hline Pmax & $\mathrm{mm} / \mathrm{d}$ & 0.0 & 10 & Maximum percolation to the groundwater \\
\hline
\end{tabular}


Table S2. Water balance and constitutive relations applied in the FLEX model

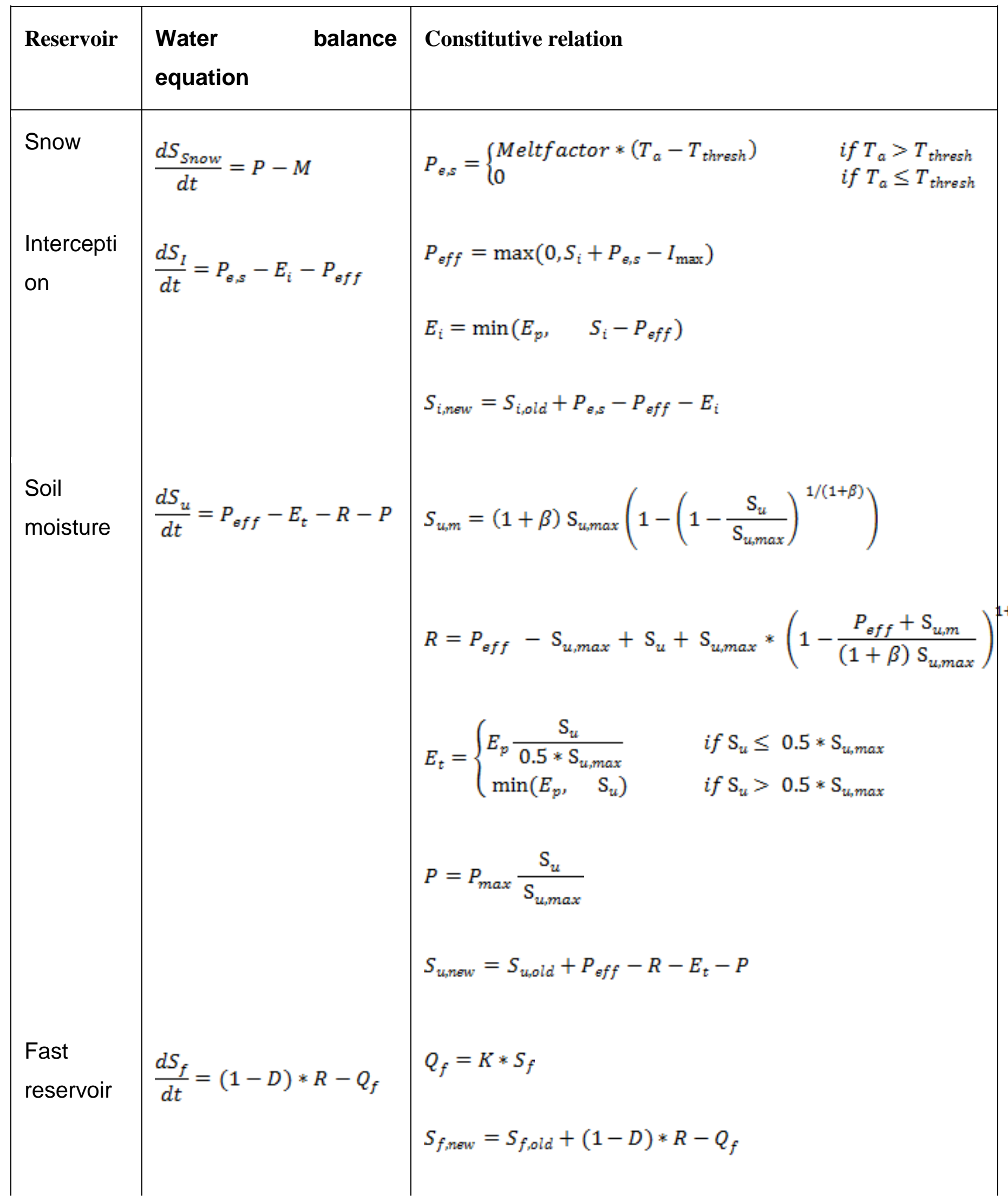




\begin{tabular}{|l|l|l|} 
Slow & $\frac{d S_{s}}{d t}=D * R+P-Q_{s}$ & $Q_{s}=K * S_{s}$ \\
reservoir & $S_{s, \text { mew }}=S_{s_{i} \text { IId }}+D * R-Q_{s}$ \\
\hline
\end{tabular}




\subsection{HYPE}

$$
\begin{aligned}
& \text { States: } \\
& \begin{array}{ll}
\text { S } & =\text { Snow } \\
\text { S1 } & =\text { Soil layer } 1 \\
\text { S2 } & =\text { Soil layer } 2 \\
\text { S3 } & =\text { Soil layer } 3
\end{array}
\end{aligned}
$$

Fluxes:

$$
\mathrm{P} \quad=\text { Precipitation }
$$

$\mathrm{Pr} \quad=$ Rainfall

$\mathrm{M}$ = Snowmelt

$\mathrm{E}_{1} \quad=$ Evaporation layer 1

$\mathrm{E}_{2} \quad=$ Evaporation layer 2

$\mathrm{qr}, \mathrm{i}=$ Surface runoff (low infiltration)

$\mathrm{qr}=$ Surface runoff

qm = Macropore flow

$\mathrm{qp}=$ Percolation

q1 = Runoff layer 1

q2 = Runofflayer 2

qs = Groundwater flow

qtot $=$ Final outflow after routing

\section{S}

$$
\mathrm{P}_{\mathrm{r}}+\mathrm{M}
$$

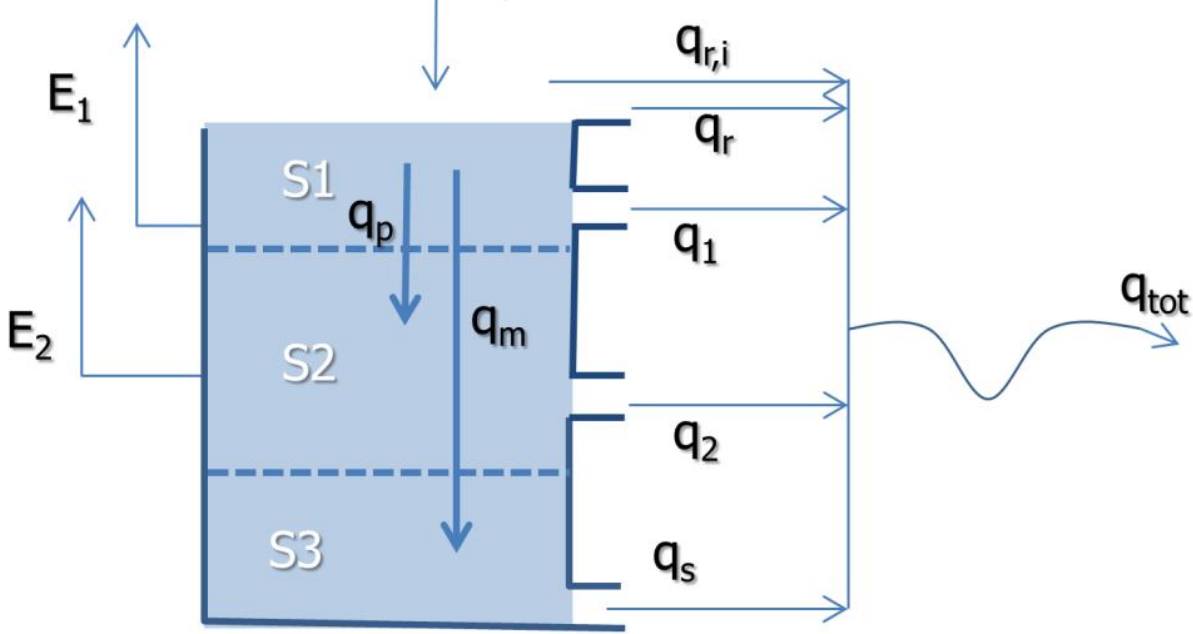

Figure S2. HYPE model structure. 
Table S2. Parameters with descriptions and prior ranges for the HYPE model

\begin{tabular}{|c|c|c|c|c|}
\hline Parameters & Unit & Min & Max & Description \\
\hline wcfc & - & 0.05 & 0.35 & $\begin{array}{l}\text { fraction of soil available for evapotranspiration but } \\
\text { not for runoff, same for all soil layers }\end{array}$ \\
\hline wcep & - & 0.05 & 0.4 & $\begin{array}{l}\text { effective porosity as a fraction, same for all soil } \\
\text { layers }\end{array}$ \\
\hline wcwp & - & 0.05 & 0.5 & wilting point as a fraction, same for all soil layers \\
\hline $\operatorname{rrcs} 1$ & - & 0.05 & 0.6 & recession coefficient for uppermost soil layer \\
\hline $\operatorname{rrcs} 2$ & - & 0.05 & 0.6 & recession coefficient for lowest soil layer \\
\hline mperc1 & - & 5 & 100 & $\begin{array}{l}\text { maximum percolation capacity from soil layer } 1 \text { to } \\
\text { soil layer } 2\end{array}$ \\
\hline mperc2 & - & 5 & 100 & $\begin{array}{l}\text { maximum percolation capacity from soil layer } 2 \text { to } \\
\text { soil layer } 3\end{array}$ \\
\hline mactrsm & - & 0.2 & 0.9 & $\begin{array}{l}\text { threshold soil water for macro-pore flow and } \\
\text { surface runoff }\end{array}$ \\
\hline macrate & - & 0.1 & 0.5 & fraction for macro-pore flow \\
\hline mactrinf & $\mathrm{mm} / \mathrm{d}$ & 10 & 45 & threshold for macro-pore flow \\
\hline srres & - & 0.01 & 0.2 & recession coefficient for surface runoff \\
\hline cmlt & $\mathrm{mm} /{ }^{\circ} / \mathrm{d}$ & 2 & 5 & melting parameter for snow \\
\hline ttmp & ${ }^{\circ} \mathrm{C}$ & 0 & 1 & $\begin{array}{l}\text { threshold temperature for snow melt and } \\
\text { evapotranspiration }\end{array}$ \\
\hline Ip & - & 0.7 & 1 & limit for potential evapotranspiration \\
\hline
\end{tabular}




$$
\begin{array}{l|l|l|l|l}
\text { rivvel } & \mathrm{m} / \mathrm{s} & 0.5 & 2 & \text { celerity of flood in watercourse }
\end{array}
$$




\subsection{TUW}

States:

$\mathrm{S}=$ Snow

Ssm = Soil moisture storage

Suz = Upper storage

$\mathrm{Slz}=$ Lower storage

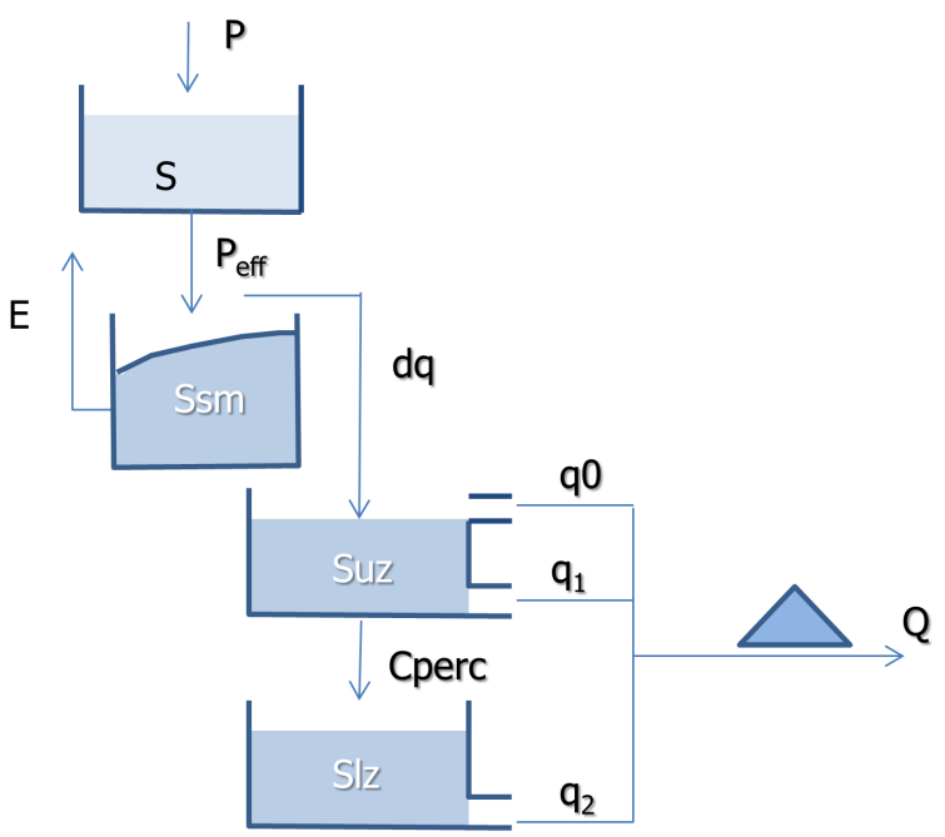

Fluxes:

$\mathrm{P}=$ Precipitation

$\mathrm{E}=$ Evaporation

Peff = Effective precipitation

Cperc $=$ Percolation

$\mathrm{q0} \quad=$ Surface runoff

$\mathrm{q}_{1}=$ Subsurface runoff

$\mathrm{q}_{2}=$ Baseflow

$\mathrm{dq}=$ Runoff

$\mathrm{Q}=$ Total discharge

Figure S3. TUW model structure. 
Table S3. Parameters with descriptions and prior ranges for the TUW model

\begin{tabular}{|c|c|c|c|c|}
\hline Parameters & Unit & Min & Max & Description \\
\hline SCF & - & 0.9 & 1.5 & Snow correction factor \\
\hline DDF & $\mathrm{Mm} /{ }^{\circ} \mathrm{C} / \mathrm{d}$ & 0 & 6 & Degree day factor \\
\hline $\operatorname{Tr}$ & $\mathrm{mm}$ & 1.0 & 3.0 & $\begin{array}{l}\text { Threshold temperature above which } \\
\text { precipitation is rain }\end{array}$ \\
\hline Ts & $\mathrm{mm}$ & -3.0 & 1 & $\begin{array}{l}\text { Threshold temperature below which } \\
\text { precipitation is snow }\end{array}$ \\
\hline $\mathrm{Tm}$ & - & -2.0 & 2 & Threshold temperature above which melt starts \\
\hline LPrat & days & 0 & 1 & $\begin{array}{l}\text { Parameter related to the limit for potential } \\
\text { evaporation }\end{array}$ \\
\hline FC & $\mathrm{mm}$ & 0.0 & 1000 & field capacity, i.e., max soil moisture storage \\
\hline Beta & - & 0.0 & 20.0 & the non linear parameter for runoff production \\
\hline k0 & days & 0.0 & 2 & storage coefficient for very fast response \\
\hline $\mathrm{k} 1$ & days & 2.0 & 5 & storage coefficient for fast response \\
\hline $\mathrm{k} 2$ & days & 5.0 & 30 & storage coefficient for slow response \\
\hline lsuz & $\mathrm{mm}$ & 1.0 & 100.0 & $\begin{array}{l}\text { threshold storage state, i.e., the very fast } \\
\text { response start if exceeded }\end{array}$ \\
\hline Cperc & $\mathrm{mm} / \mathrm{d}$ & 0.0 & 8.0 & constant percolation rate \\
\hline bmax & days & 0.0 & 10.0 & maximum base at low flows \\
\hline croute & day $2 / \mathrm{mm}$ & 0.0 & 50.0 & free scaling parameter \\
\hline
\end{tabular}




\subsection{HYMOD}

States:

$\mathrm{S}=$ Snow

$\mathrm{Su}=$ Soil moisture storage

$\mathrm{Sq}=$ Quick storage

Ss = Slow storage
Fluxes:

$\mathrm{P} \quad=$ Precipitation

$\mathrm{E}=$ Evaporation

Peff = Effective precipitation

$Q q=$ Quick flow

Qs = Slow flow

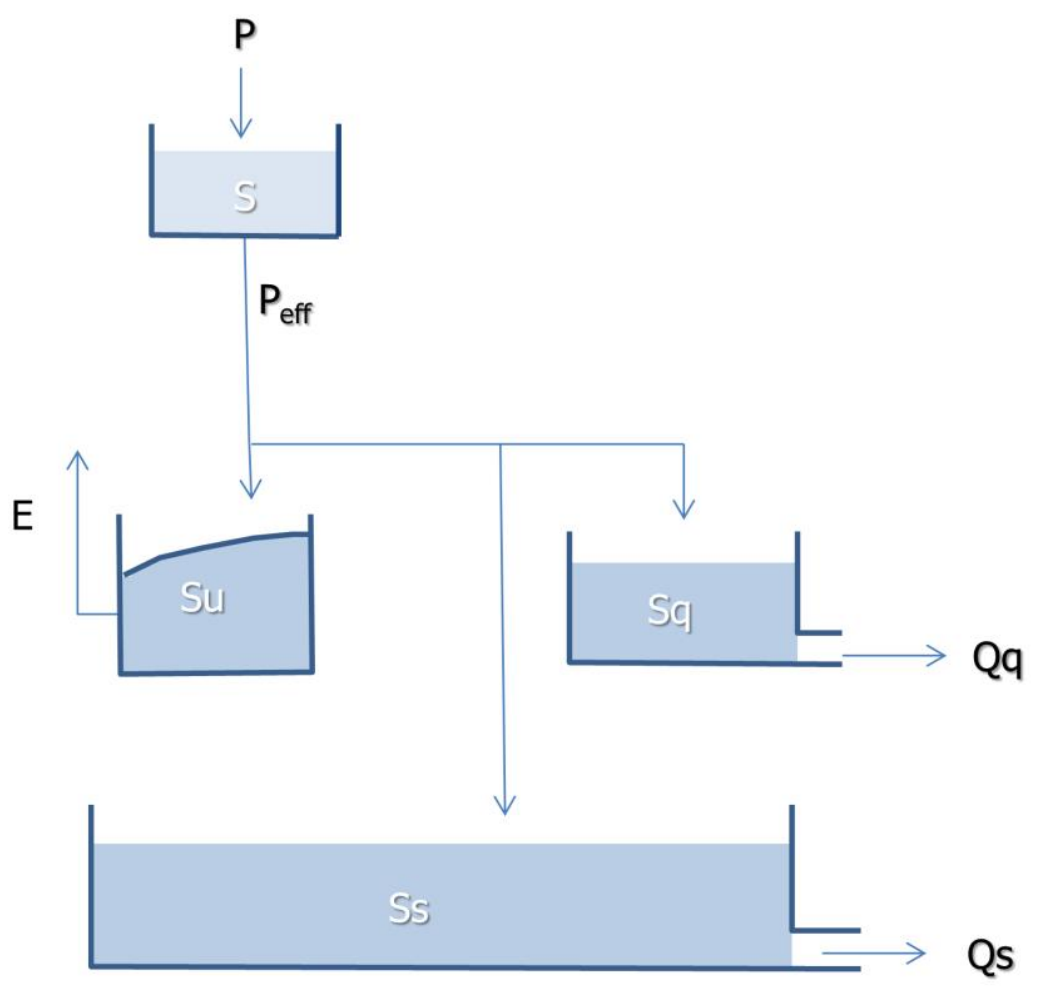

Figure S4. HYMOD model structure. 
Table S4. Parameters with descriptions and prior ranges for the HYMOD model

\begin{tabular}{|c|c|c|c|c|}
\hline Parameters & Unit & Min & Max & Description \\
\hline DDF & $\mathrm{Mm} /{ }^{\circ} \mathrm{C} / \mathrm{d}$ & 0 & 6 & Degree day factor \\
\hline $\mathrm{Tb}$ & ${ }^{\circ} \mathrm{C}$ & -3.0 & 3.0 & $\begin{array}{l}\text { Threshold temperature above which } \\
\text { precipitation is rain }\end{array}$ \\
\hline Tth & ${ }^{\circ} \mathrm{C}$ & -2.0 & 2 & $\begin{array}{l}\text { Threshold temperature below which } \\
\text { precipitation is snow }\end{array}$ \\
\hline alpha & - & 0.0 & 1 & $\begin{array}{l}\text { Parameter determining separation between fast } \\
\text { and slow runoff }\end{array}$ \\
\hline Cmax & $\mathrm{Mm}$ & 0 & 1000 & max soil moisture storage \\
\hline Beta & - & 0.01 & 3.0 & the non linear parameter for runoff production \\
\hline $\mathrm{Kq}$ & Days & 1.0 & 3.0 & storage coefficient for fast response \\
\hline Ks & Days & 1.0 & 20 & storage coefficient for slow response \\
\hline
\end{tabular}




\section{S3. Calibration results}

Table S5. Feasible number of model runs for HJ Andrews WS1

\begin{tabular}{|c|r|r|r|r|}
\hline HJ Andrews & FLEX & TUW & HYPE & HYMOD \\
\hline Window 1 & 653 & 18448 & 9017 & 23809 \\
\hline Window 2 & 4146 & 17692 & 9419 & 46291 \\
\hline Window 3 & 1433 & 6129 & 9663 & 9334 \\
\hline Window 4 & 7023 & 30599 & 9609 & 46917 \\
\hline Window 5 & 252 & 15862 & 9922 & 26773 \\
\hline Window 6 & 6 & 843 & 9990 & 1356 \\
\hline Window 7 & 100 & 12194 & 9876 & 27186 \\
\hline Window 8 & 116 & 13415 & 10000 & 24778 \\
\hline Window 9 & 8 & 1437 & 9969 & 1965 \\
\hline Window 10 & 35 & 12626 & 9367 & 12073 \\
\hline Window 11 & 3 & 5242 & 9996 & 3158 \\
\hline Window 12 & 149 & 30344 & 9597 & 30321 \\
\hline Window 13 & 54 & 20485 & 9427 & 19171 \\
\hline Window 14 & 47 & 12567 & 9625 & 15843 \\
\hline
\end{tabular}

Table S6. Feasible number of model runs for Hubbard Brook WS2

\begin{tabular}{|c|r|r|r|r|}
\hline Hubbard Brook WS2 & FLEX & TUW & HYPE & HYMOD \\
\hline Window 1 & 48 & 38741 & 19977 & 9765 \\
\hline Window 2 & 2717 & 5798 & 15678 & 4456 \\
\hline Window 3 & 7258 & 8295 & 19961 & 11825 \\
\hline Window 4 & 1031 & 5184 & 19445 & 8276 \\
\hline Window 5 & 3496 & 51959 & 19940 & 21003 \\
\hline Window 6 & 247 & 18393 & 17674 & 6683 \\
\hline Window 7 & 137 & 11248 & 19999 & 4491 \\
\hline Window 8 & 18 & 22928 & 19708 & 1665 \\
\hline Window 9 & 108 & 37971 & 19914 & 18884 \\
\hline Window 10 & 25 & 35596 & 19961 & 3085 \\
\hline Window 11 & 19 & 33985 & 19997 & 11258 \\
\hline Window 12 & 107 & 21985 & 19604 & 2749 \\
\hline Window 13 & 34 & 31767 & 19999 & 7932 \\
\hline Window 14 & 26 & 6450 & 19938 & 6999 \\
\hline Window 15 & 66 & 31713 & 19999 & 9296 \\
\hline Window 16 & 77 & 37002 & 19999 & 11879 \\
\hline Window 17 & 29 & 14256 & 19940 & 6031 \\
\hline Window 18 & 162 & 32063 & 19841 & 10672 \\
\hline Window 19 & 67 & 45893 & 19995 & 8326 \\
\hline Window 20 & 19 & 27436 & 20000 & 10652 \\
\hline Window 21 & 49 & 7872 & 19978 & 4943 \\
\hline Window 22 & 37 & 11060 & 19994 & 6304 \\
\hline Window 23 & 1289 & 48703 & 19910 & 23705 \\
\hline
\end{tabular}


Table S7. Feasible number of model runs for Hubbard Brook WS5

\begin{tabular}{|c|r|r|r|r|}
\hline Hubbard Brook WS5 & FLEX & TUW & HYPE & HYMOD \\
\hline Window 1 & 103 & & 99980 & \\
\hline Window 2 & 194 & & 99980 & \\
\hline Window 3 & 161 & 33592 & 99980 & 16819 \\
\hline Window 4 & 204 & 28342 & 99980 & 9658 \\
\hline Window 5 & 686 & 27978 & 99980 & 6893 \\
\hline Window 6 & 32 & 2262 & 99980 & 972 \\
\hline Window 7 & 123 & 22632 & 99980 & 10496 \\
\hline Window 8 & 86 & 40841 & 99980 & 9029 \\
\hline Window 9 & 162 & 36043 & 99980 & 17378 \\
\hline Window 10 & 70 & 35020 & 99980 & 6351 \\
\hline Window 11 & 86 & 6415 & 99980 & 4034 \\
\hline Window 12 & 140 & 6054 & 99970 & 466 \\
\hline Window 13 & 311 & 44640 & 99980 & 17834 \\
\hline Window 14 & 469 & 34557 & 99980 & 16863 \\
\hline Window 15 & 40 & 2934 & 99980 & 1215 \\
\hline Window 16 & 249 & 26178 & 99980 & 9073 \\
\hline Window 17 & 85 & 11782 & 99980 & 4428 \\
\hline Window 18 & 190 & 23955 & 99980 & 7983 \\
\hline Window 19 & 242 & 44971 & 99980 & 13454 \\
\hline Window 20 & 215 & 39199 & 99980 & 16568 \\
\hline Window 21 & 59 & 7734 & 99980 & 4164 \\
\hline Window 22 & 33 & 6020 & 99980 & 3958 \\
\hline Window 23 & 1873 & 50668 & 99980 & 23343 \\
\hline
\end{tabular}



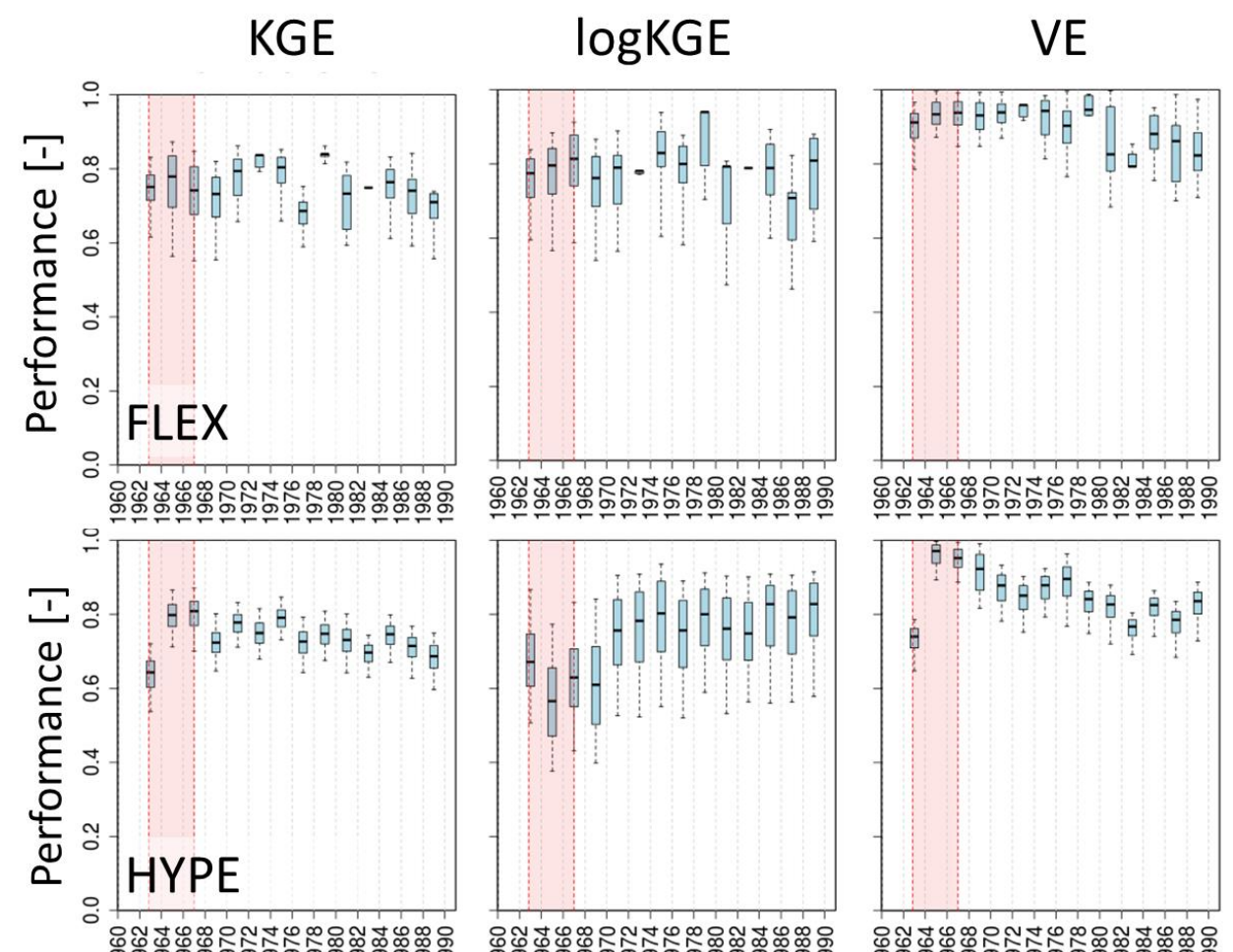

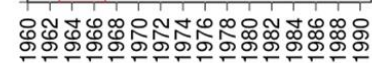
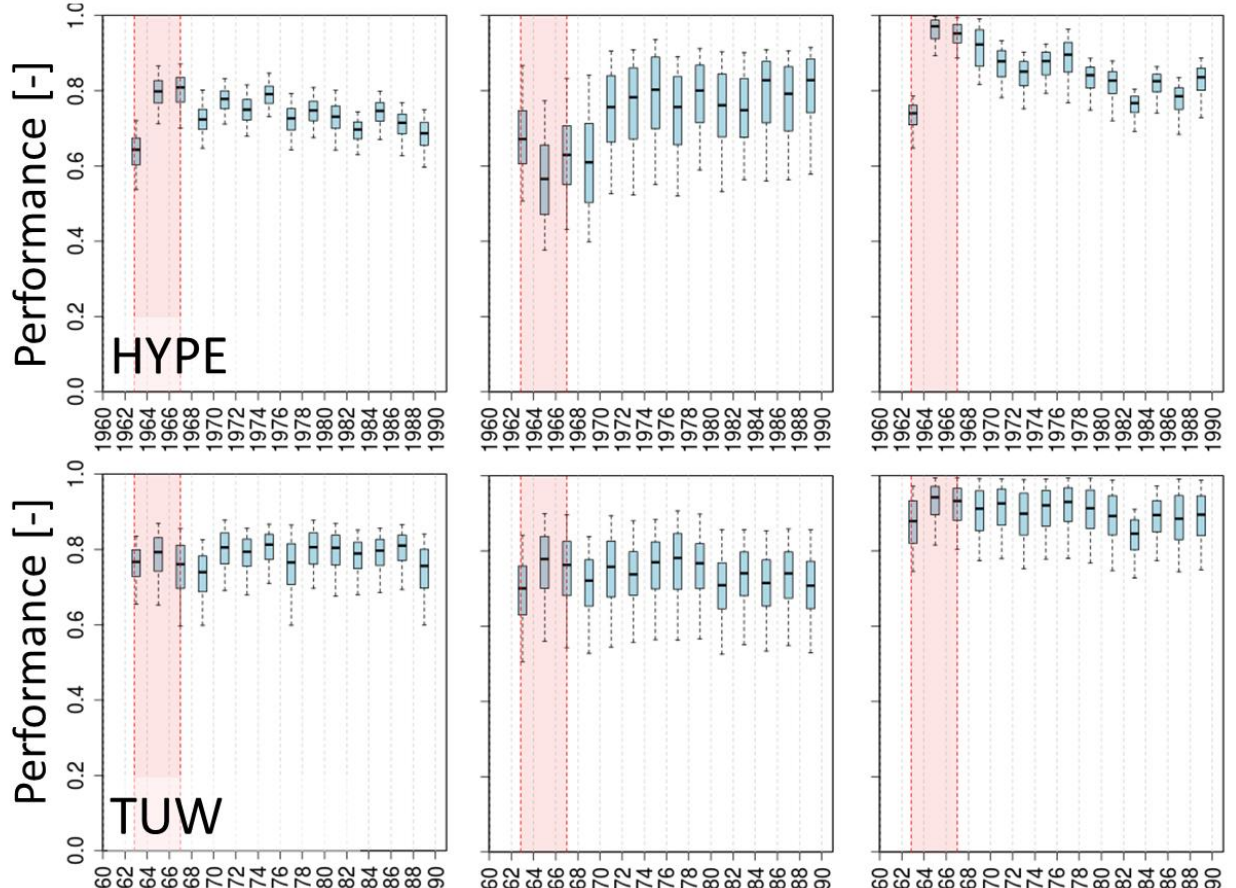

:

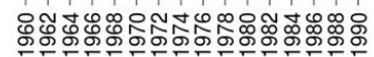
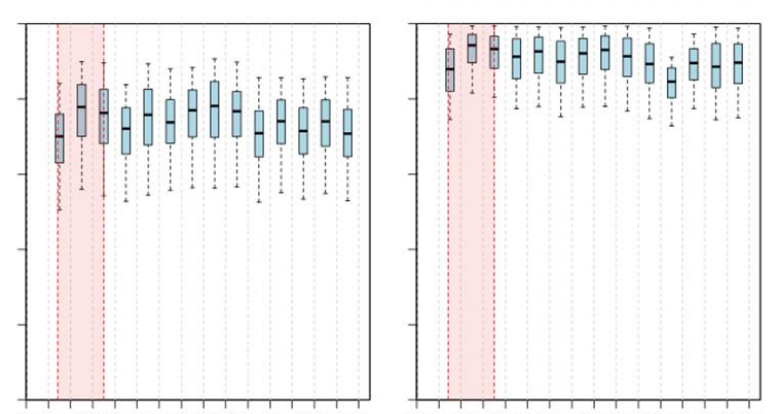

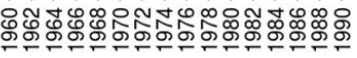

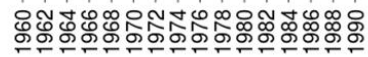
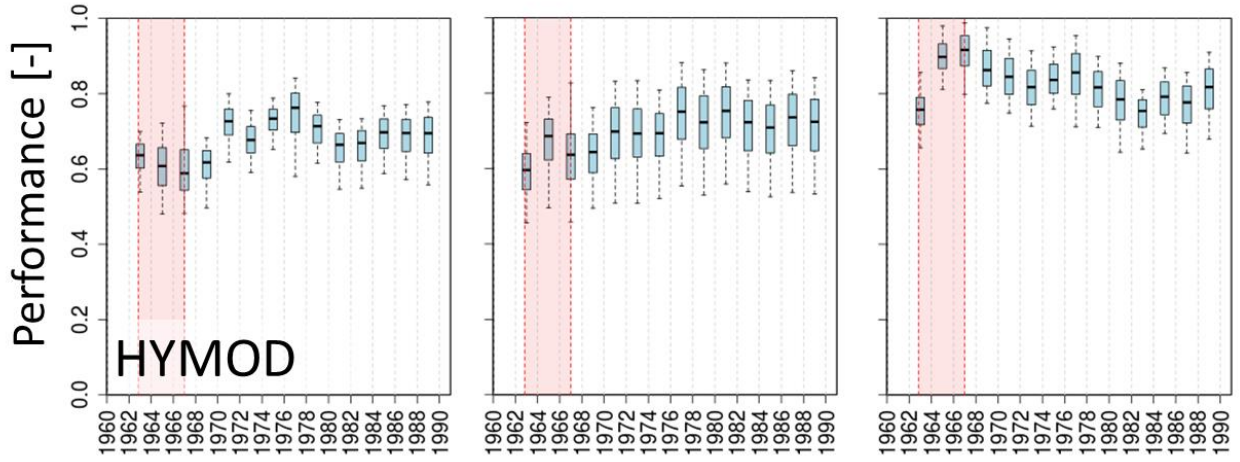

Figure S5. Objective function values of Kling-Gupta Efficiency (KGE), log Kling-Gupta Efficiency (logKGE) and volume error (VE) for HJ Andrews WS1 resulting from the calibration for each time window. 

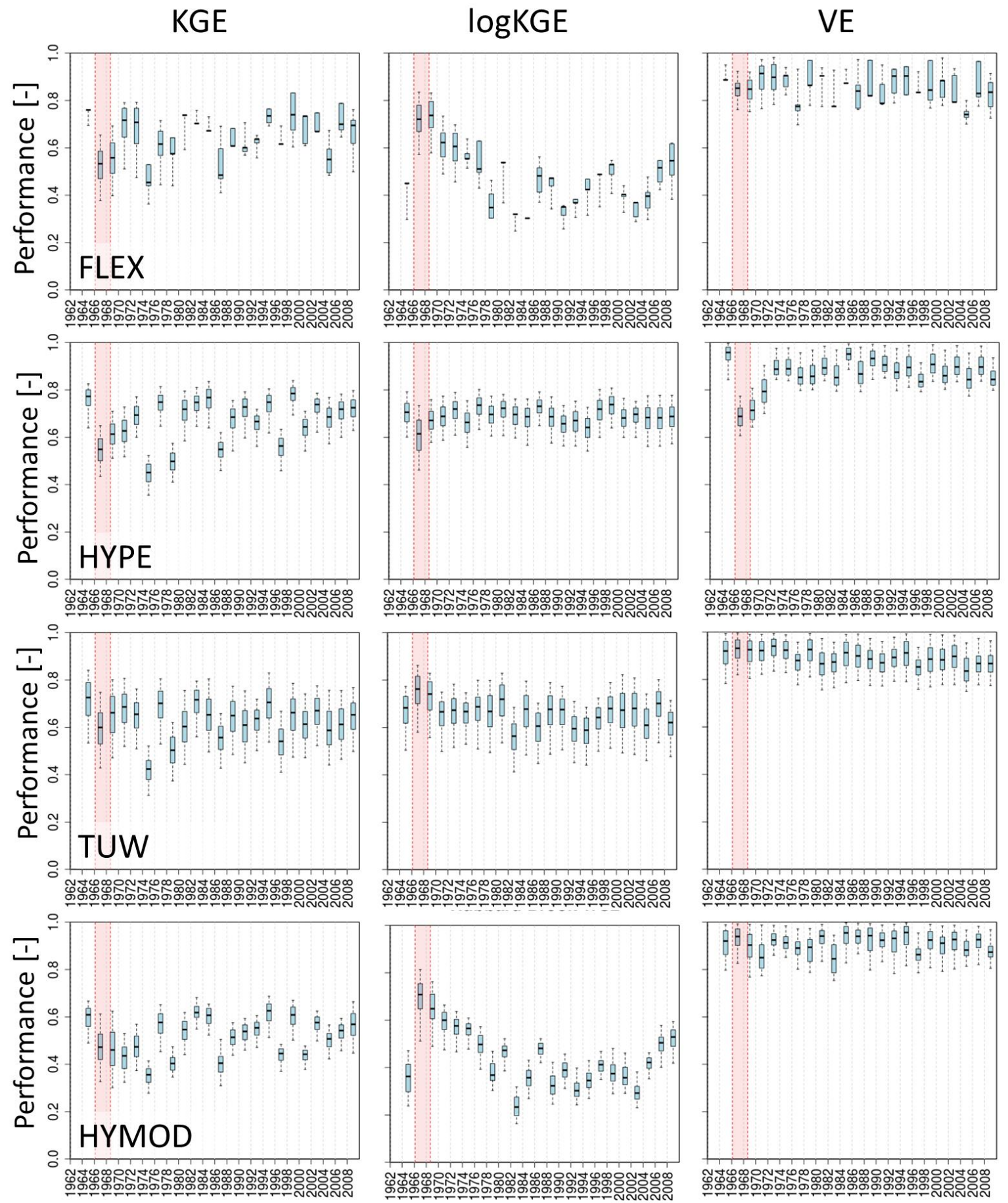

Figure S6. Objective function values of Kling-Gupta Efficiency (KGE), log Kling-Gupta Efficiency (logKGE) and volume error (VE) for Hubbard Brook WS2 resulting from the calibration for each time window. 

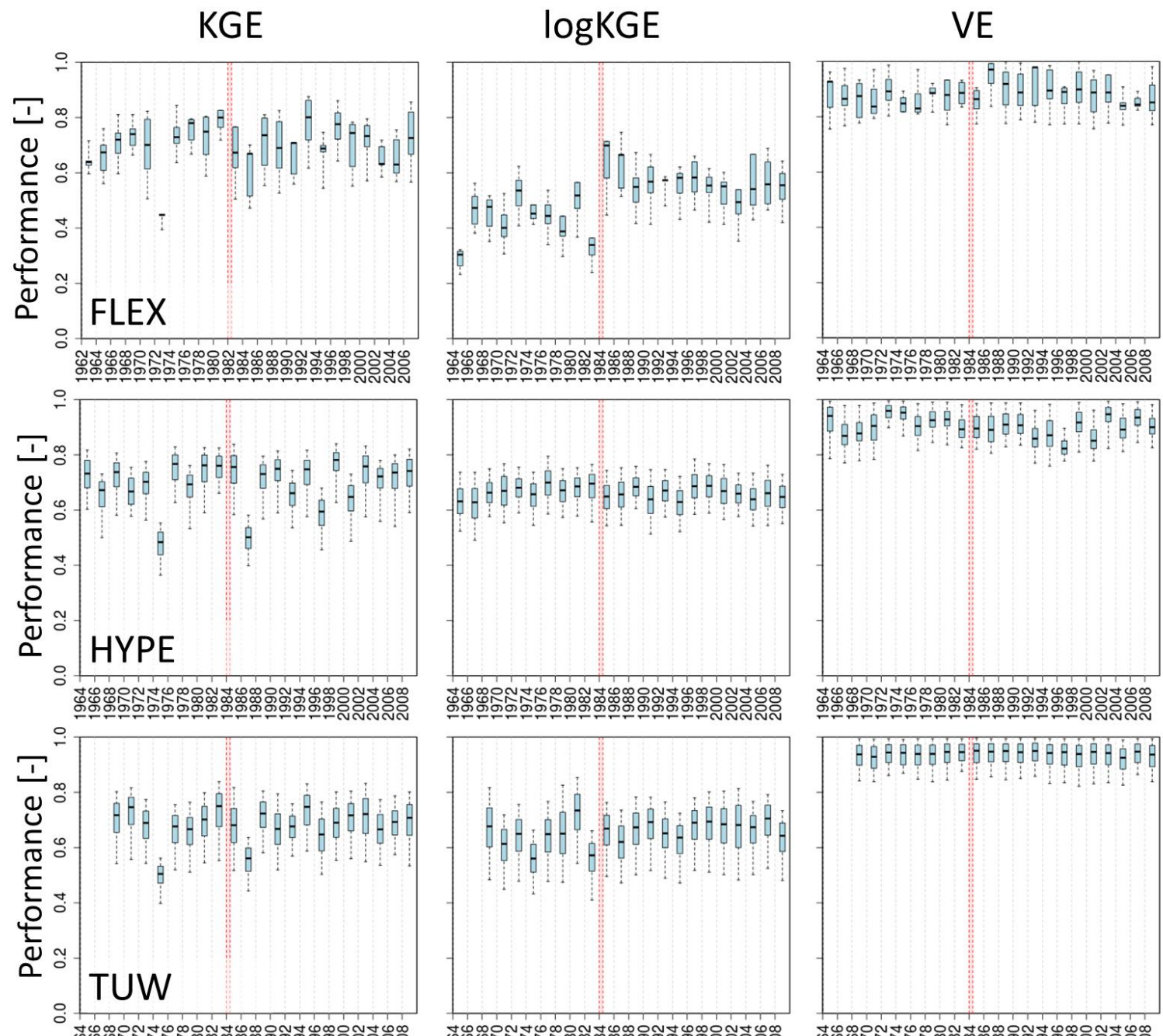

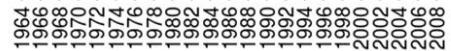

小
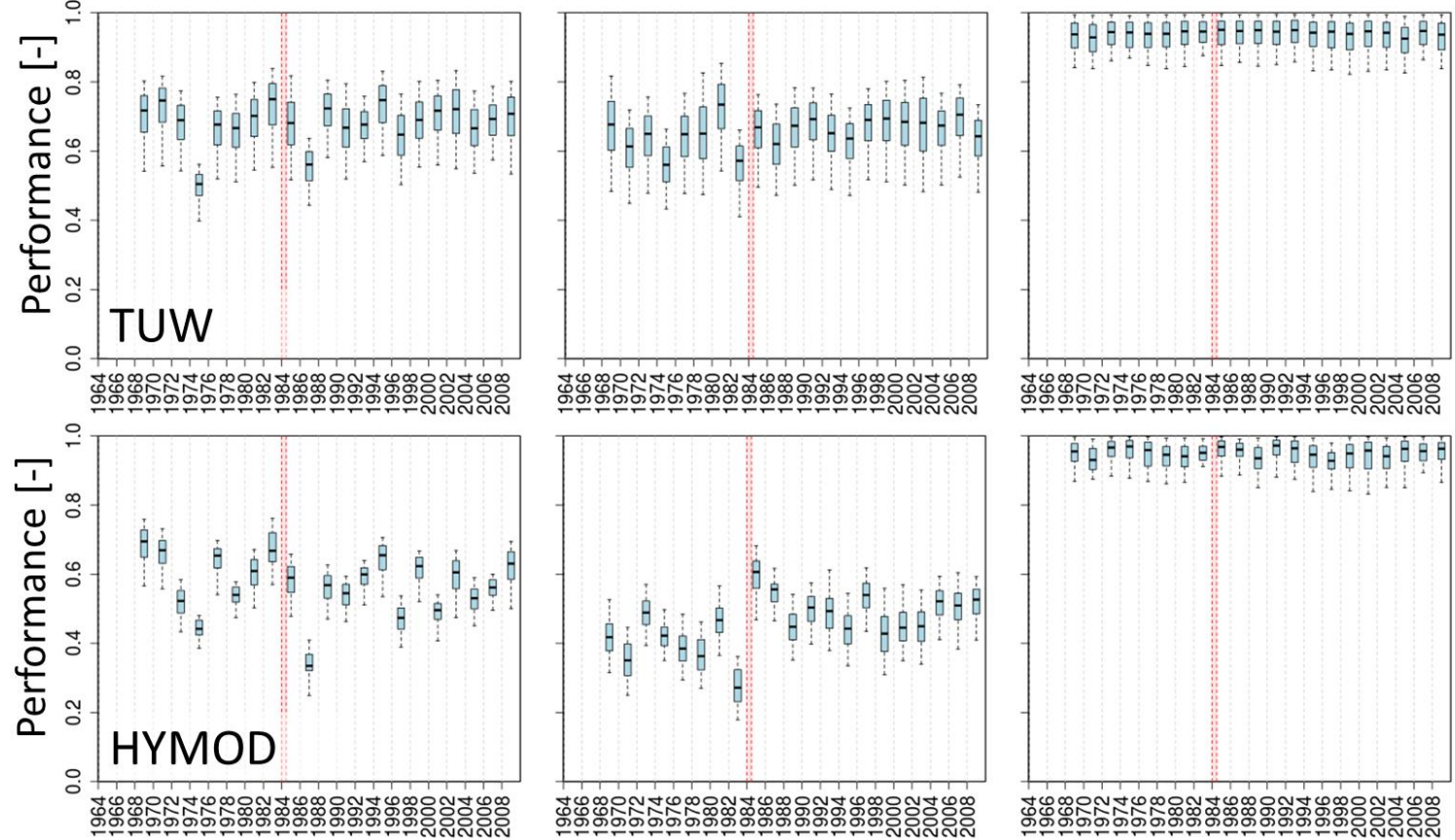

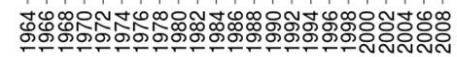

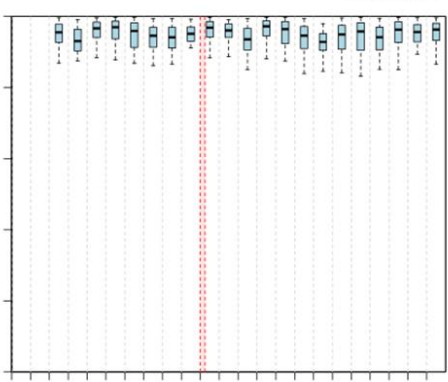

ఫ̋ڤ

Figure S7. Objective function values of Kling-Gupta Efficiency (KGE), log Kling-Gupta Efficiency (logKGE) and volume error (VE) for Hubbard Brook WS5 resulting from the calibration for each time window. 


\section{HJ Andrews WS2 Hubbard Brook WS3}
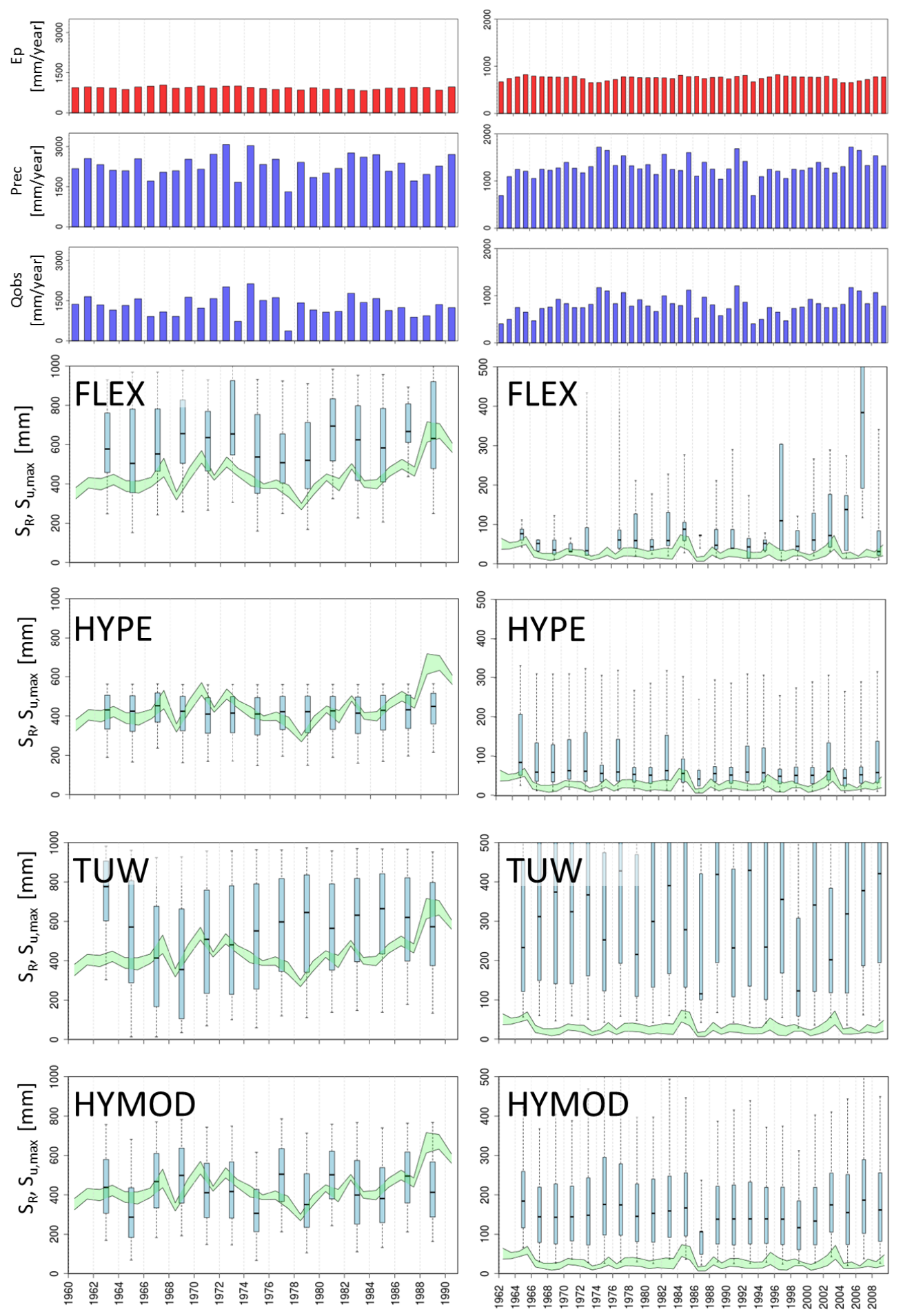

Figure S8. Evolution of root zone storage capacity $S_{\mathrm{R}, 1 \mathrm{yr}}$ from water balance-based estimation (green shaded area, a range of solutions due to the sampling of the unknown interception capacity) compared with $S_{\mathrm{u}, \max , 2 \mathrm{yr}}$ estimates obtained from the calibration of four models (FLEX, HYPE, TUW, HYMOD; blue boxplots) for a) HJ Andrews WS2, b) Hubbard Brook WS3. 


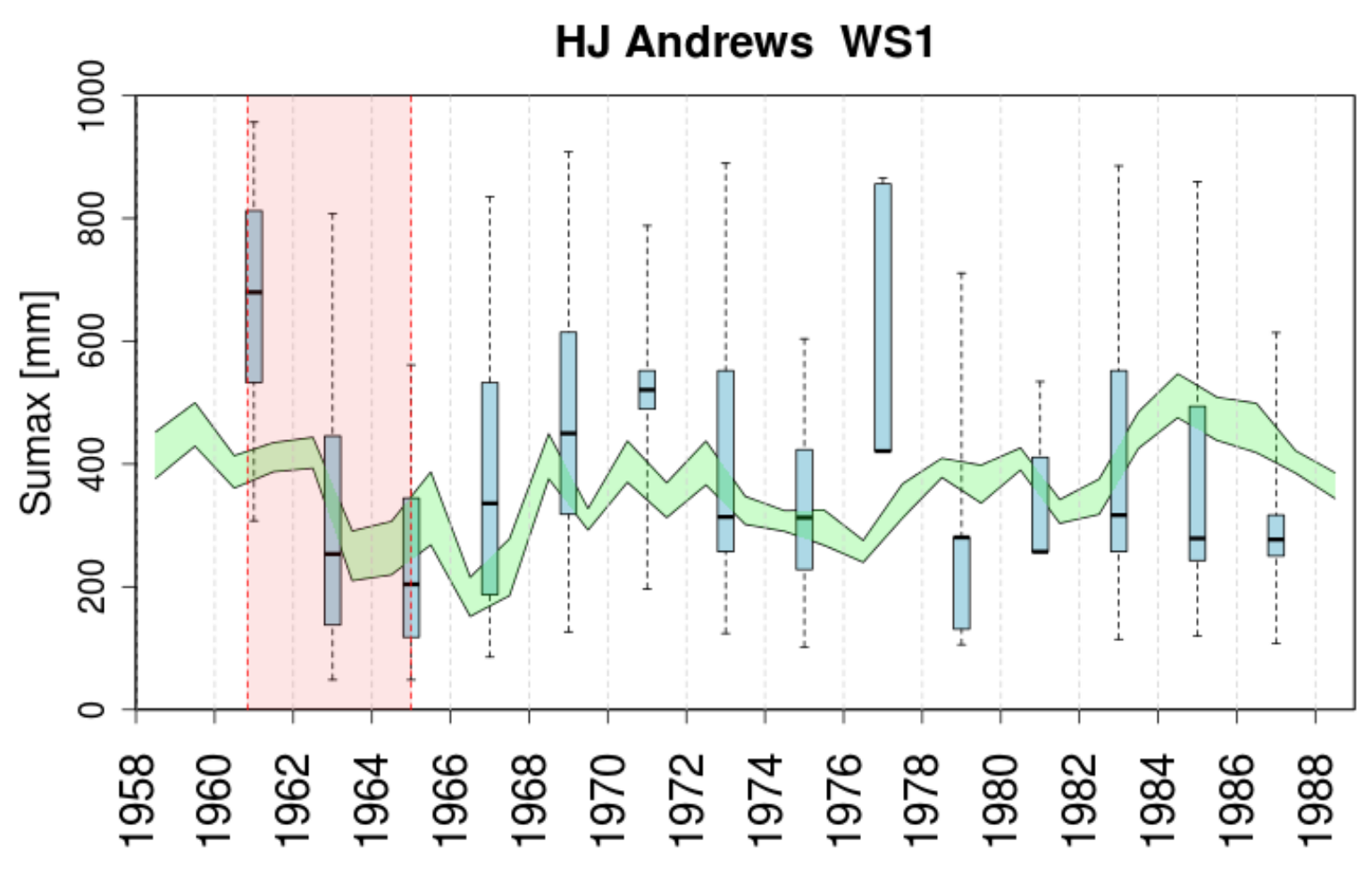

Figure 9. Evolution of root zone storage capacity $S_{R, 1 y r}$ from a 5 year water balance-based estimation (green shaded area, a range of solutions due to the sampling of the unknown interception capacity) compared with $S_{u, m a x, 2 y r}$ estimates obtained from the calibration of the FLEX model. 


\section{S4. Posterior parameter distributions}

\subsection{FLEX}
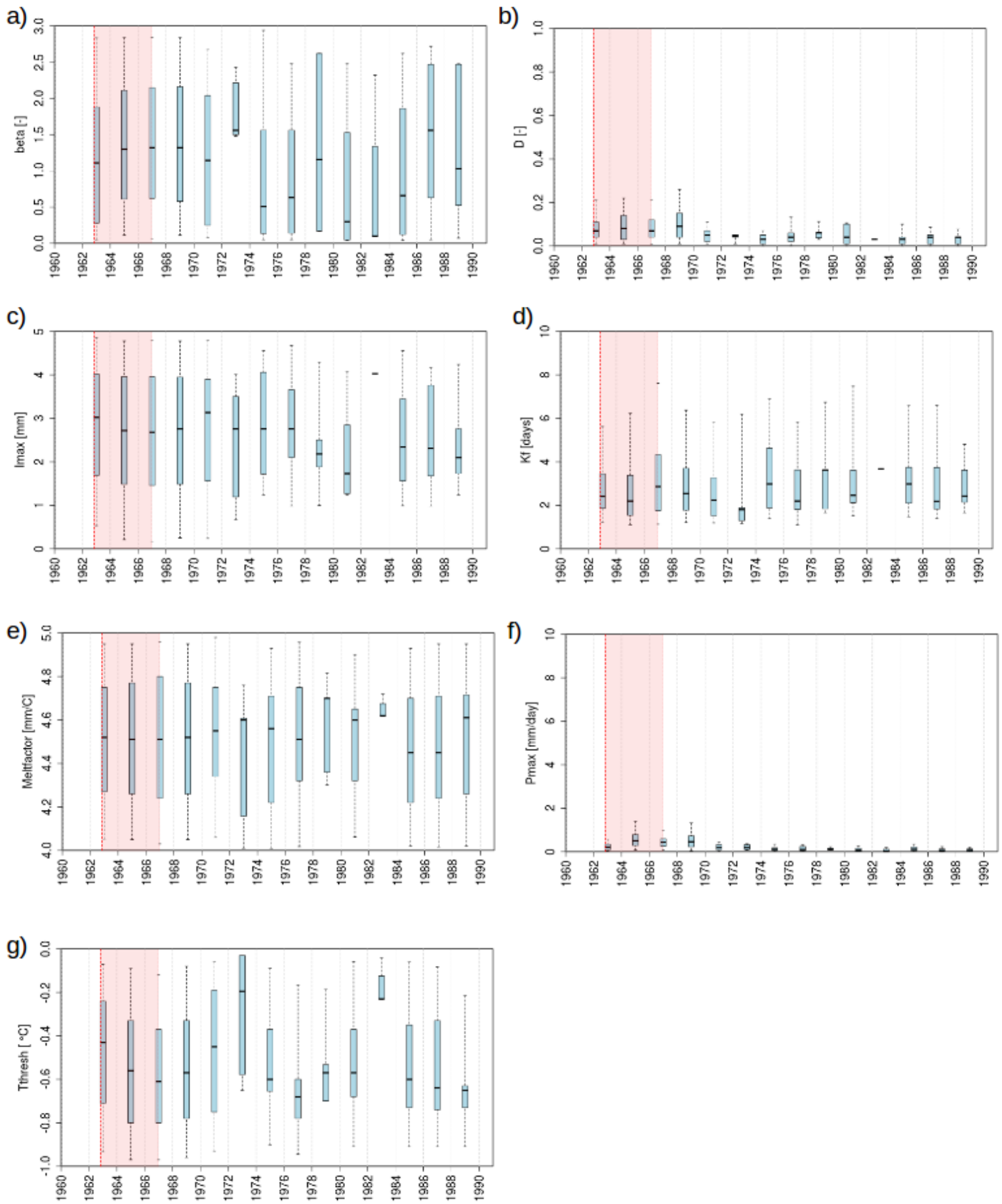

Figure S10. Posterior parameter distributions for the FLEX model in HJ Andrews WS1. 

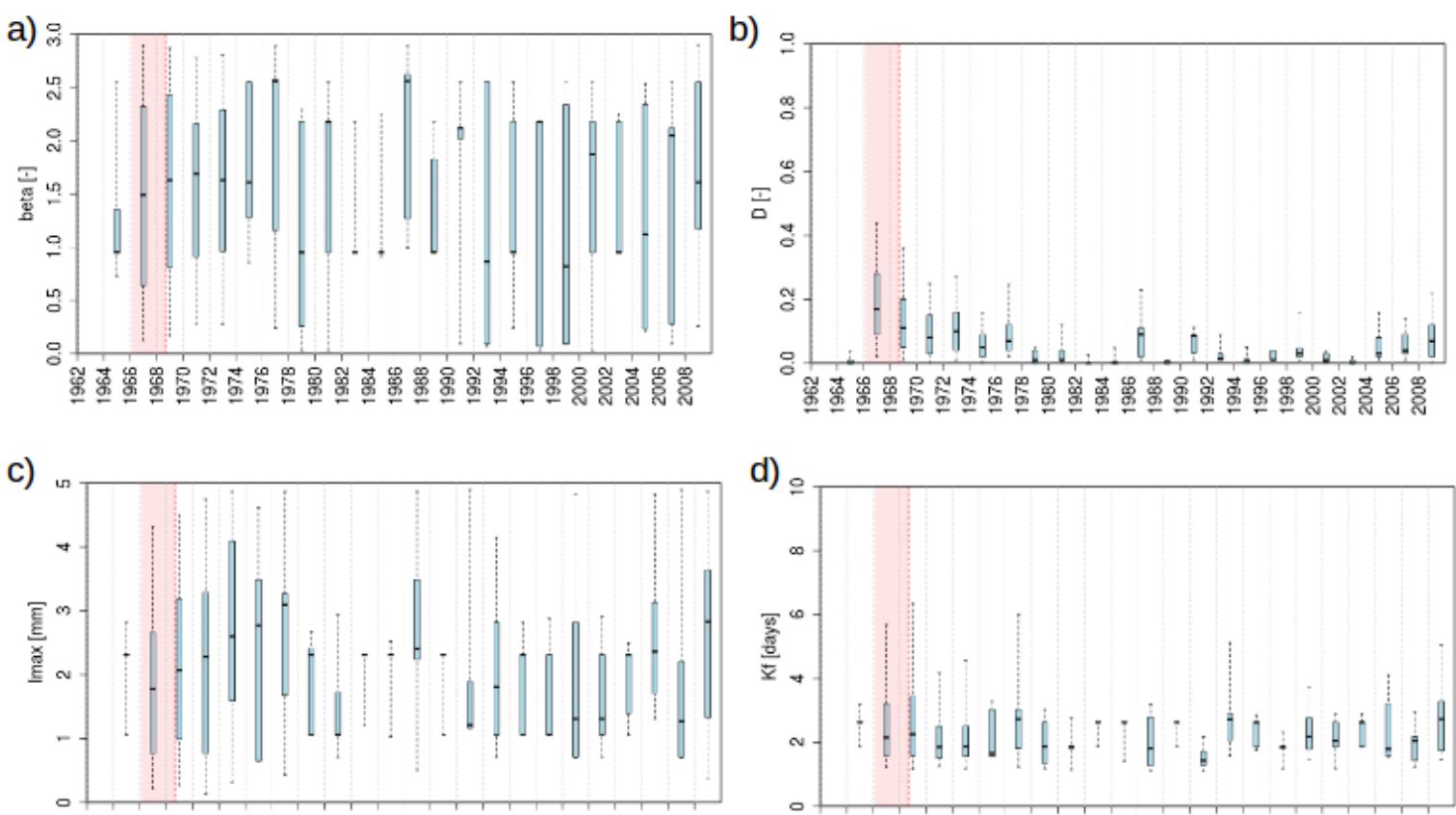

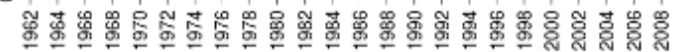
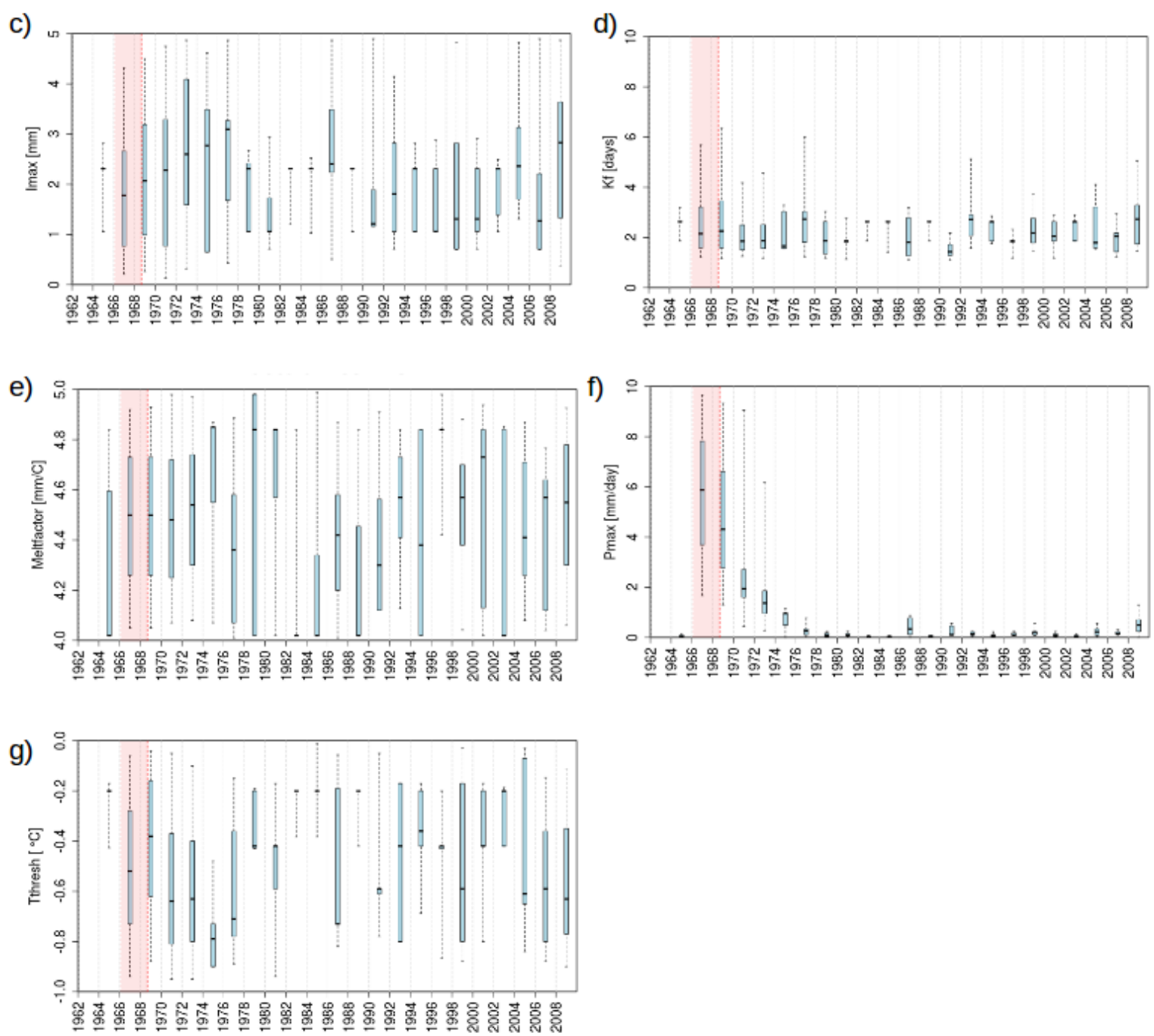

Figure S11. Posterior parameter distributions for the FLEX model in Hubbard Brook WS2. 

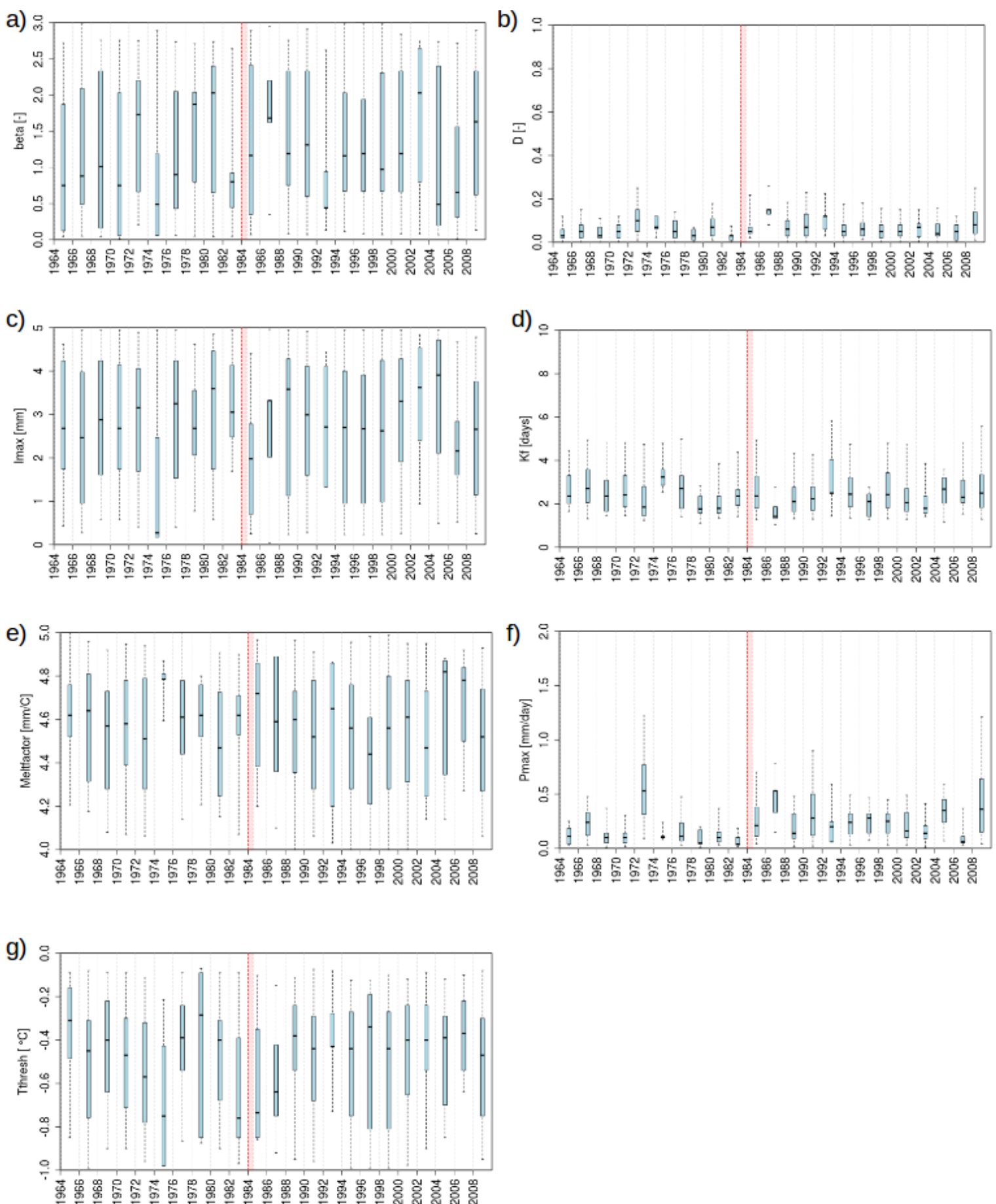

Figure S12. Posterior parameter distributions for the FLEX model in Hubbard Brook WS5. 


\subsection{HYPE}
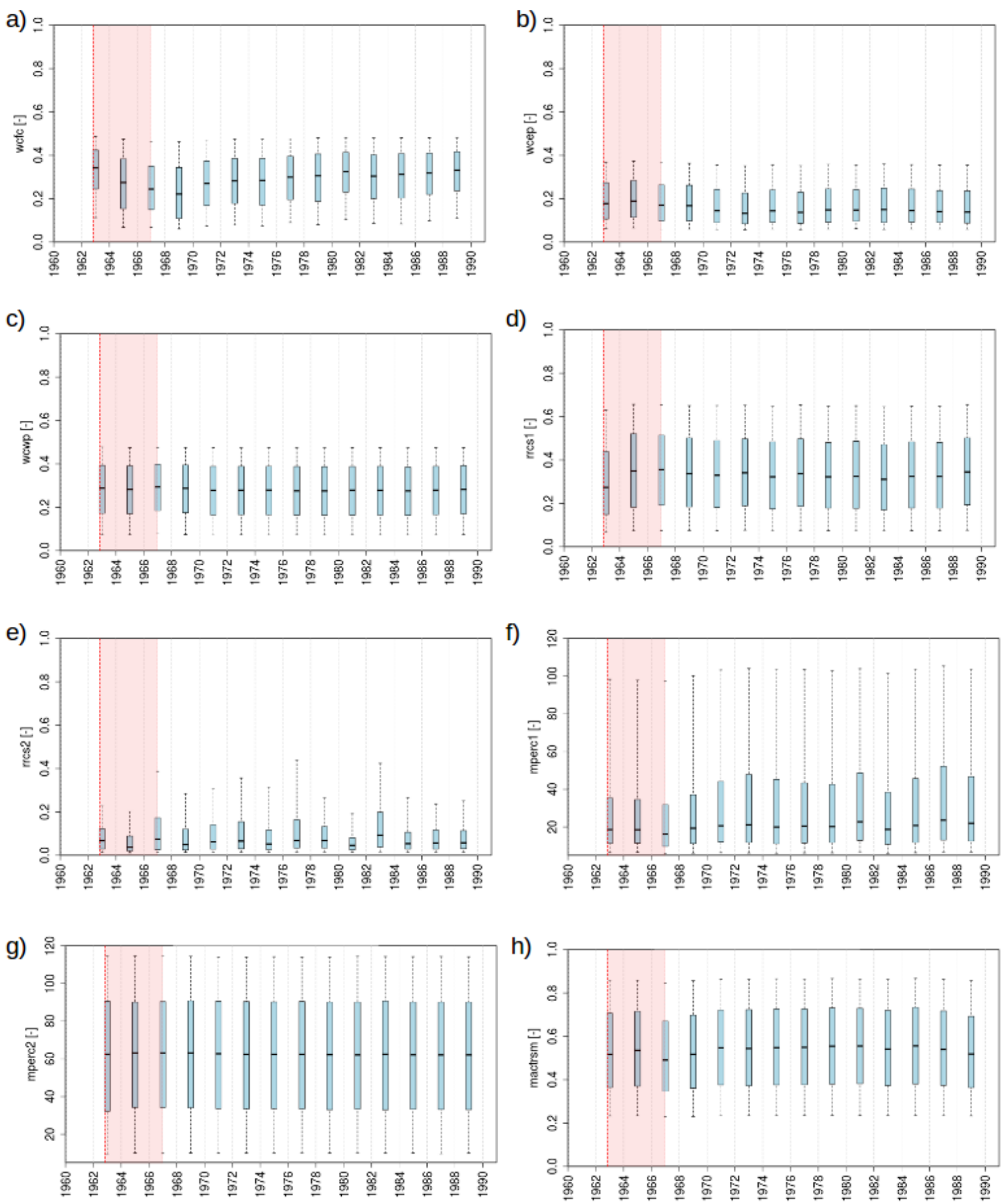

Figure S13. Posterior parameter distributions for the HYPE model in HJ Andrews WS1. 
a)

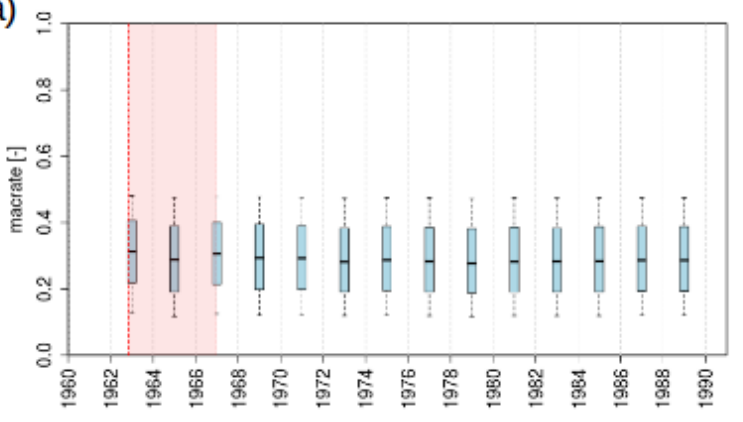

c)

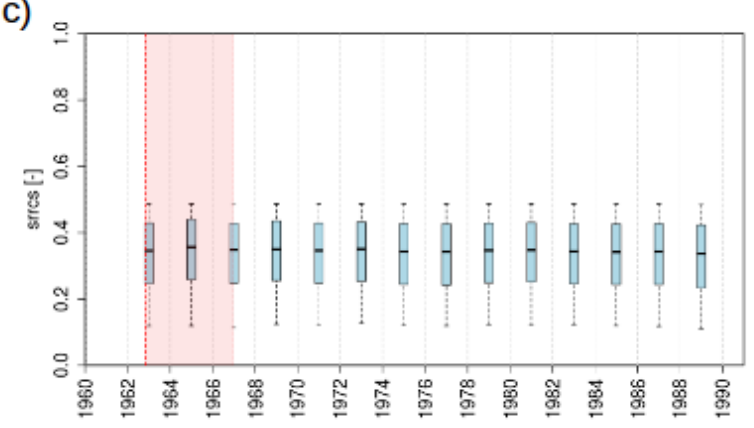

e)

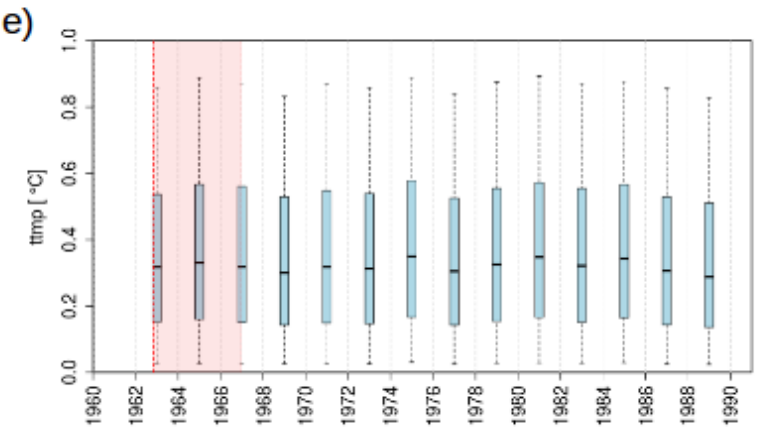

g)

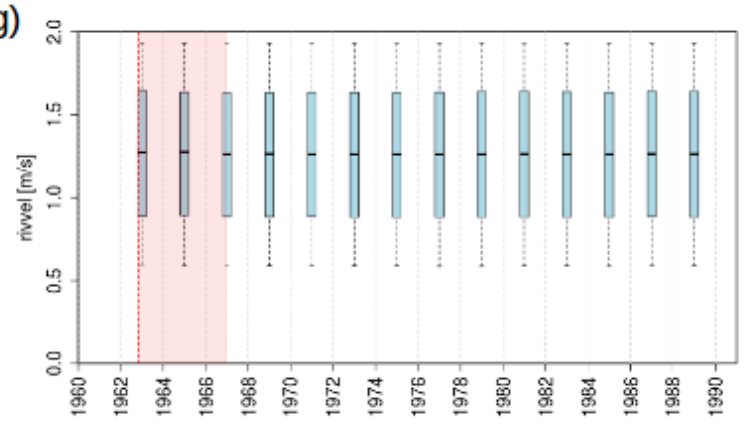

b)

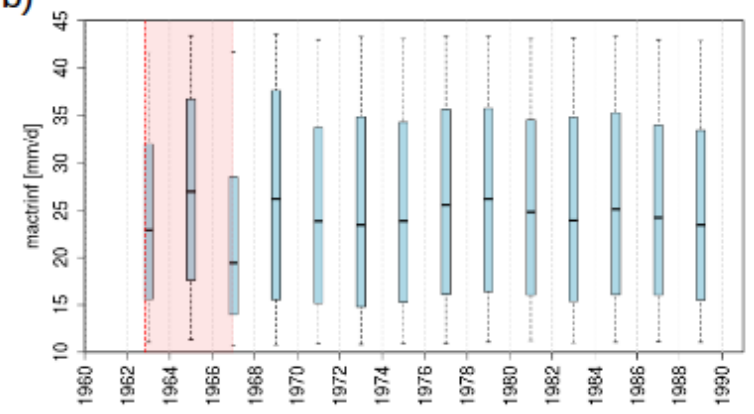

d)

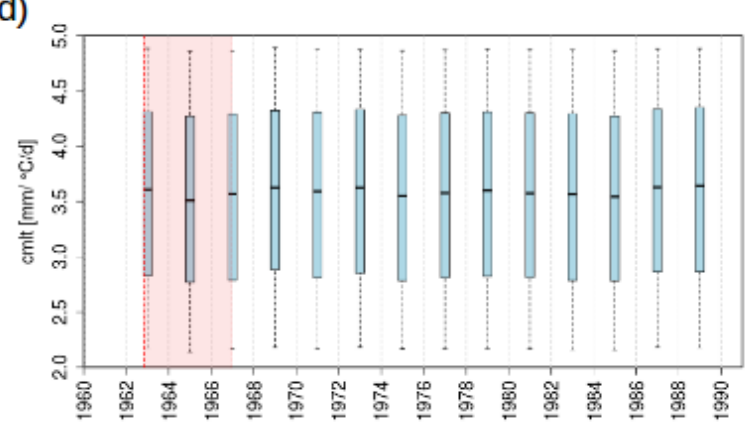

f)

Figure S14. Continued posterior parameter distributions for the HYPE model in HJ Andrews WS1. 
a)

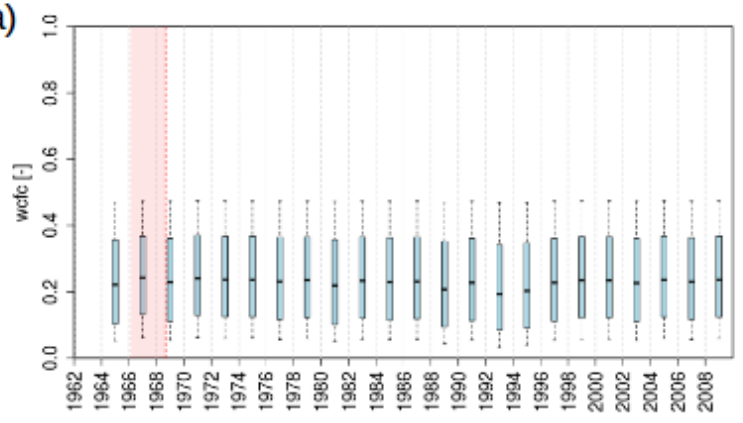

c)

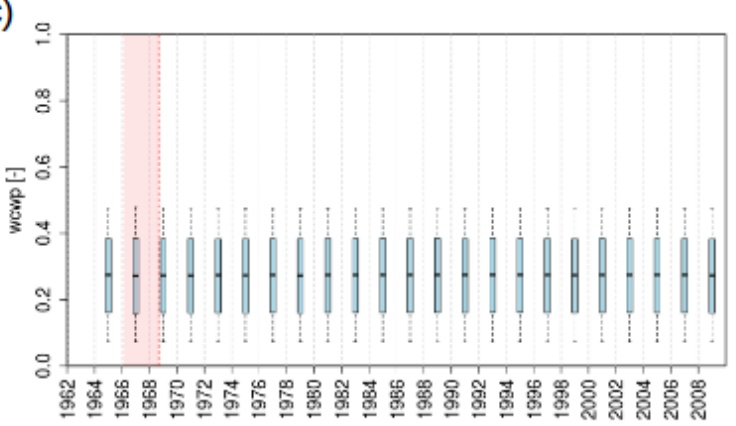

b)

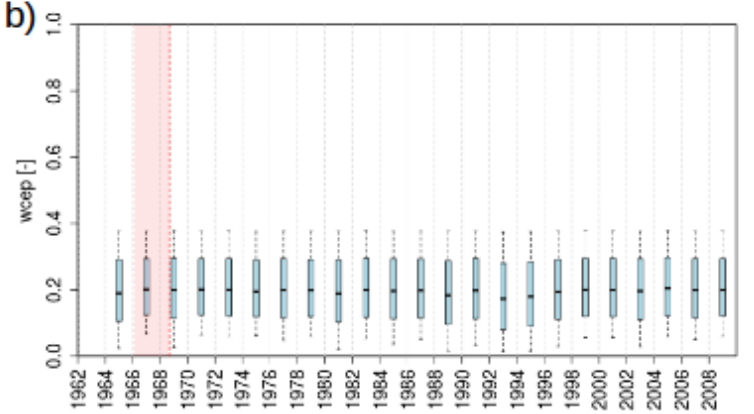

d)

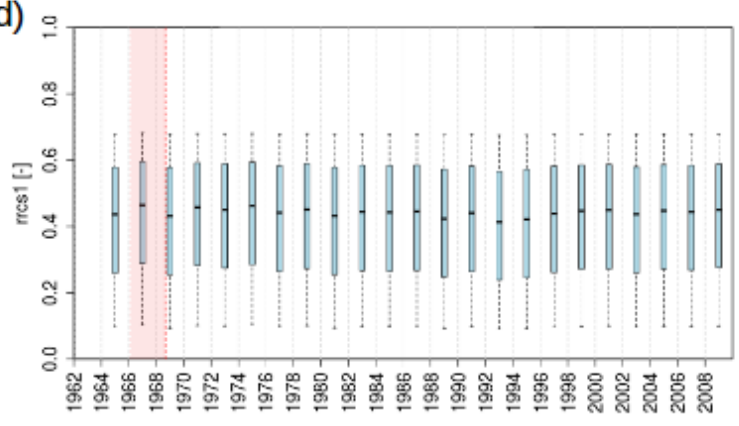

e)

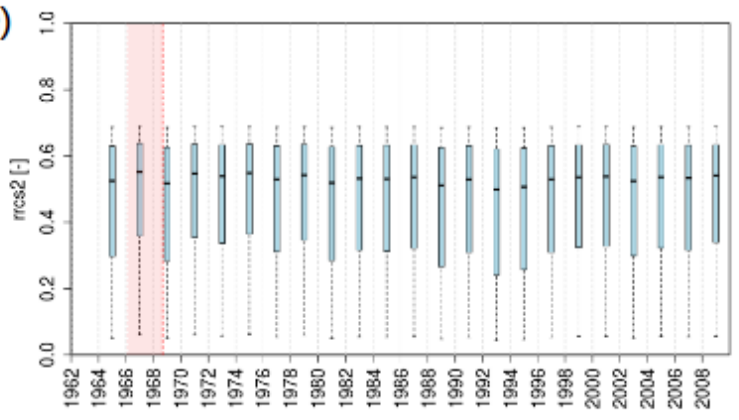

g)

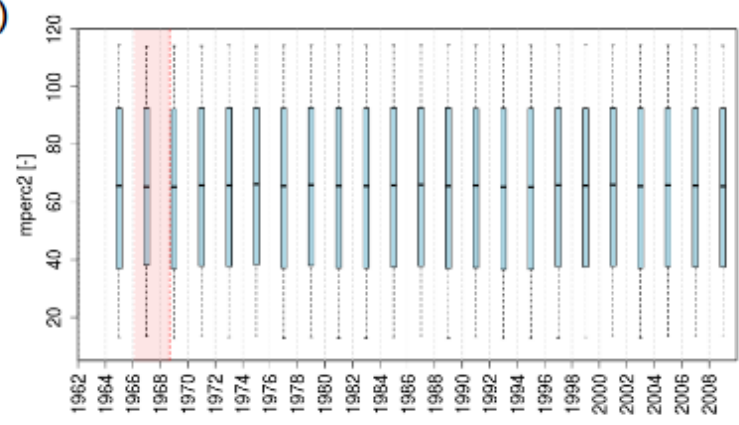

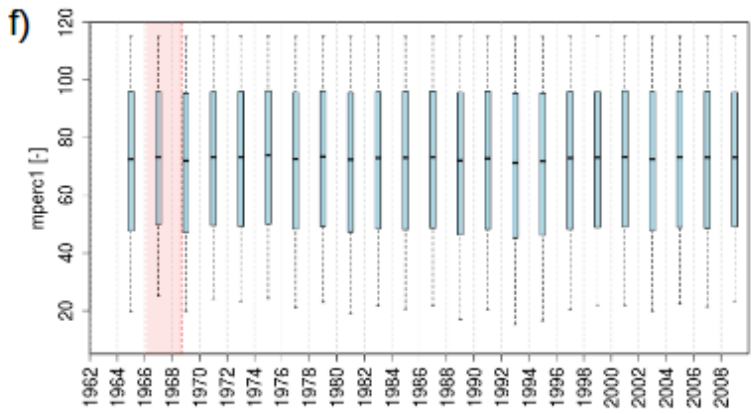

h)

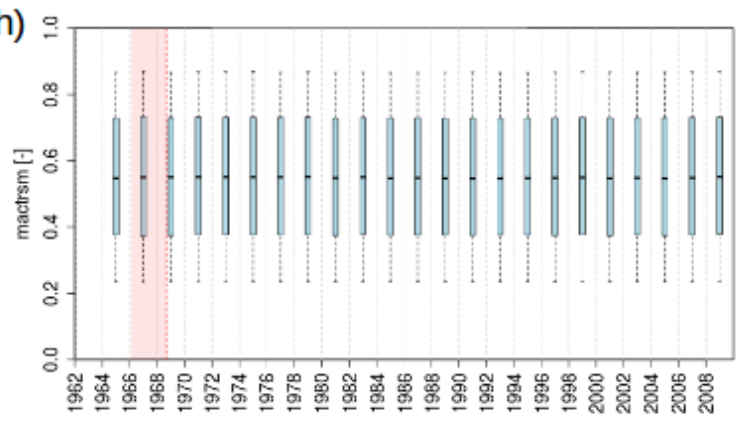

Figure S15. Posterior parameter distributions for the HYPE model in Hubbard Brook WS2. 
a)

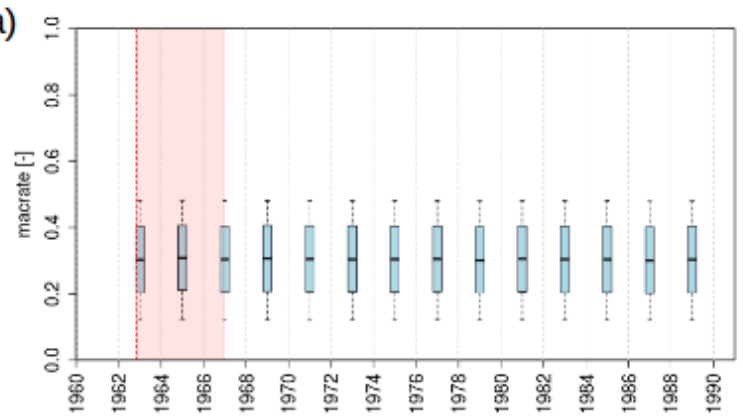

c)

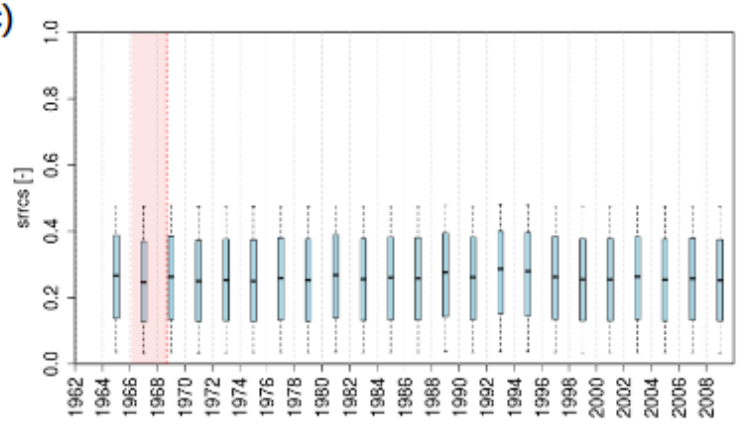

b)

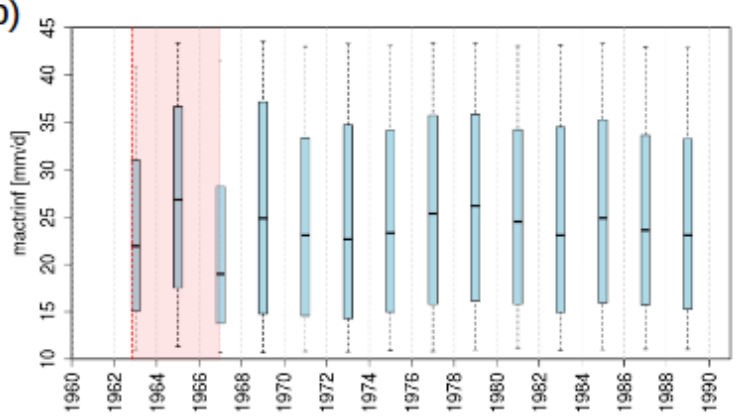

d)

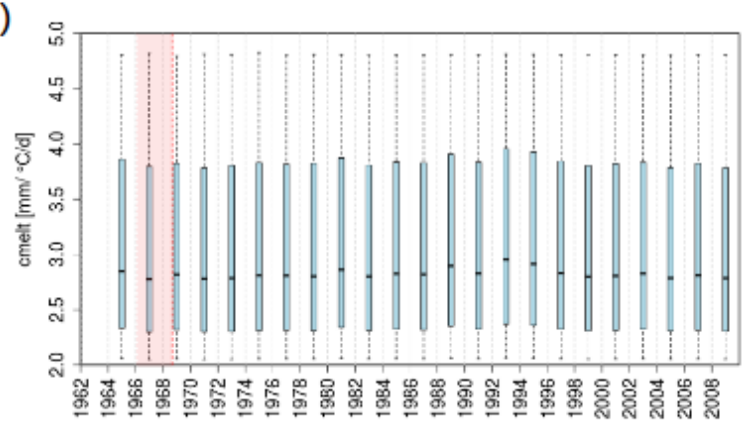

e)

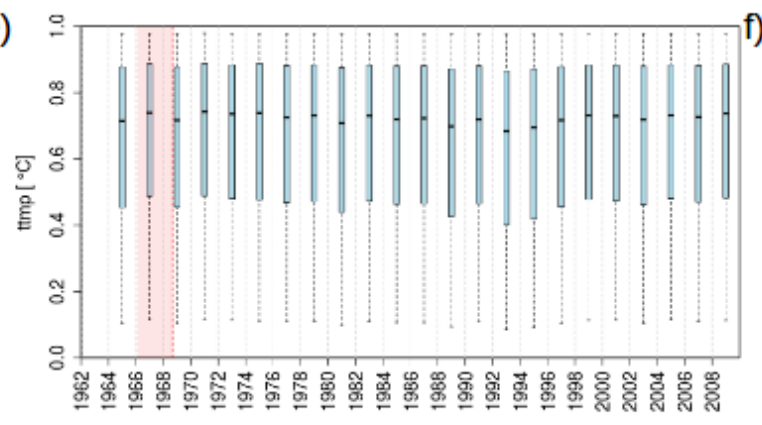

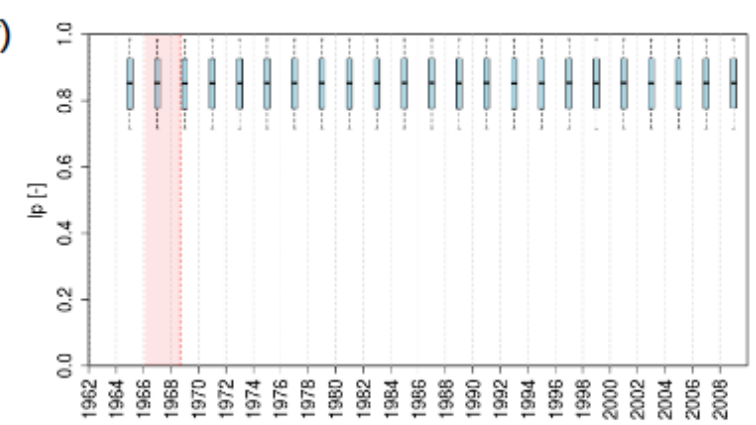

g)

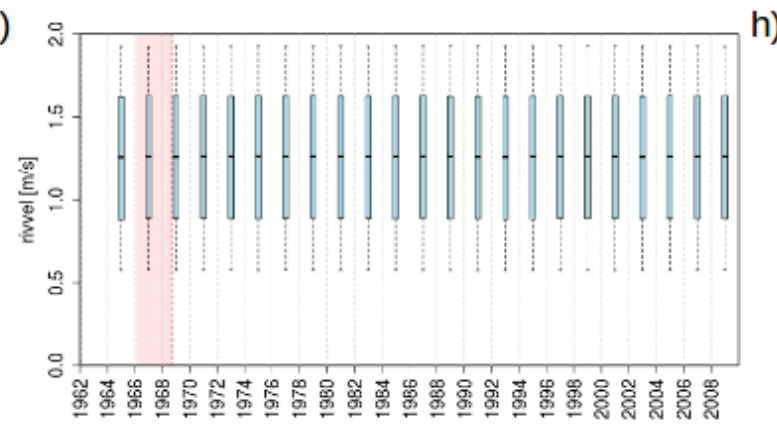

Figure S16. Continued posterior parameter distributions for the HYPE model in Hubbard Brook WS2. 
a)

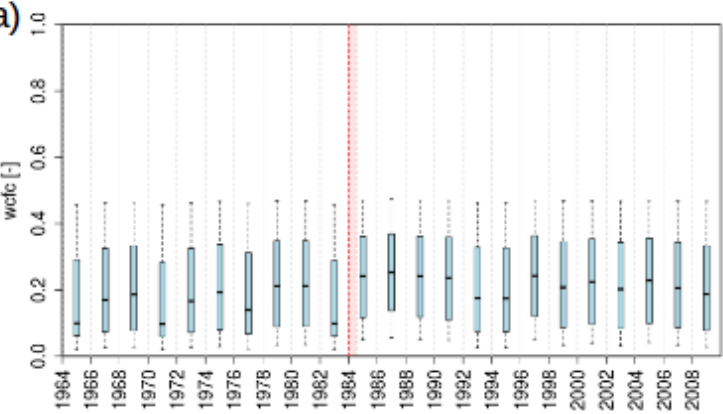

c)

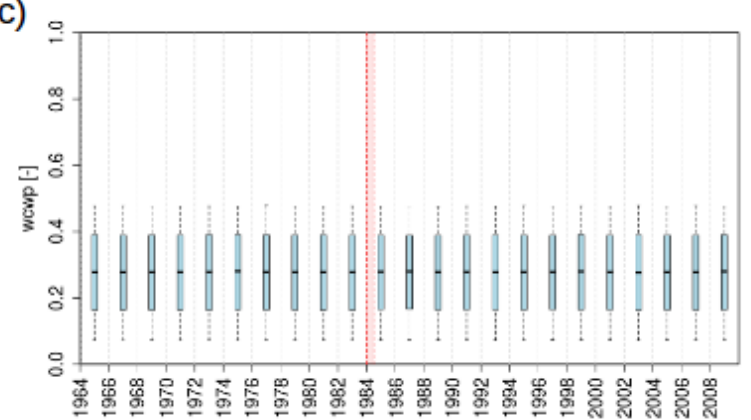

e)

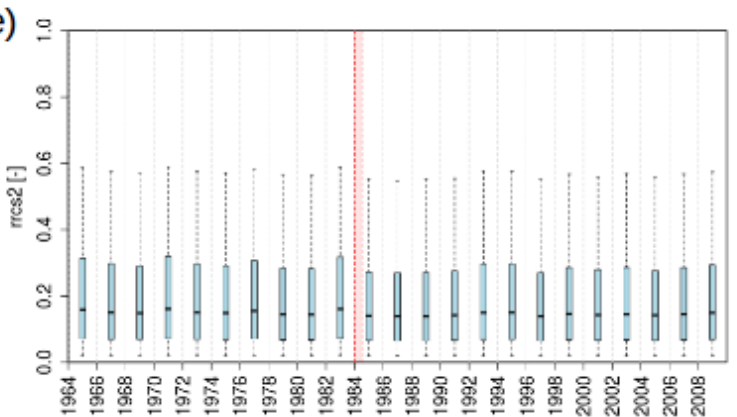

g)

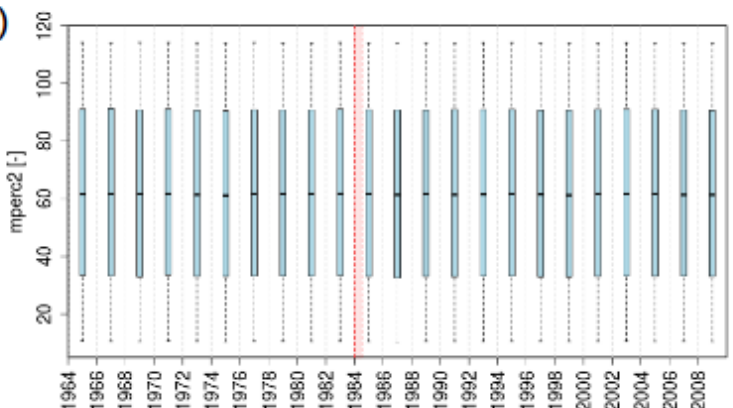

b)

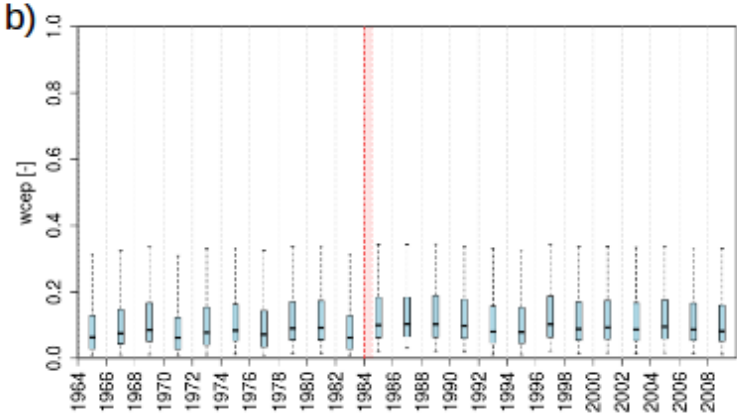

d)
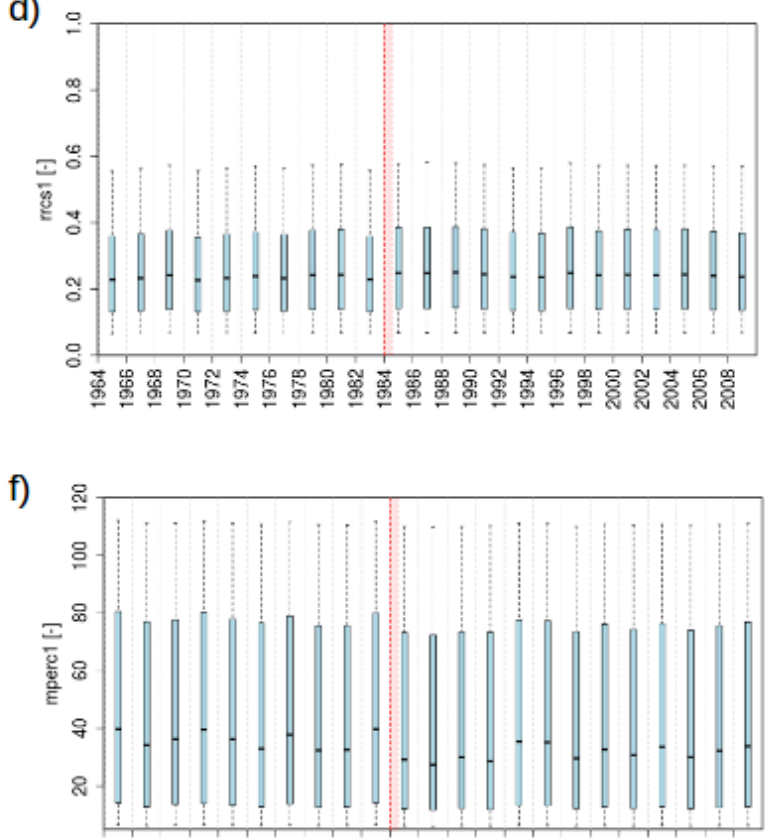

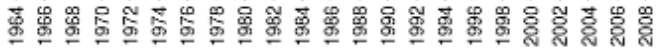

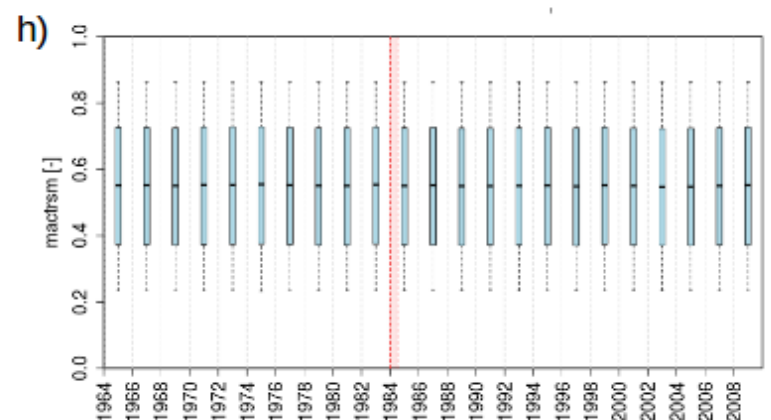

Figure S17. Posterior parameter distributions for the HYPE model in Hubbard Brook WS5. 
a)

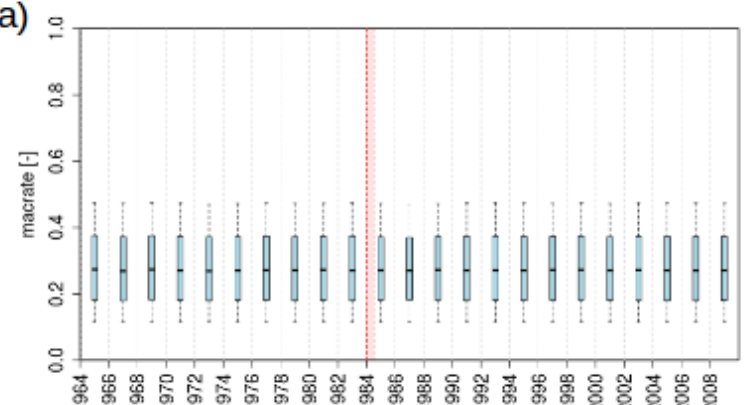

c)

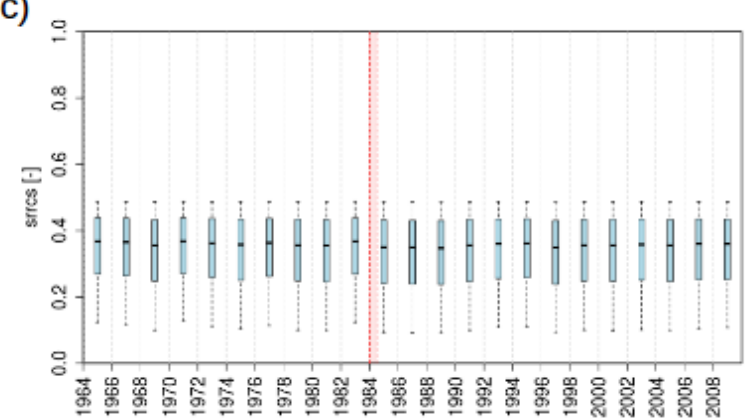

e)

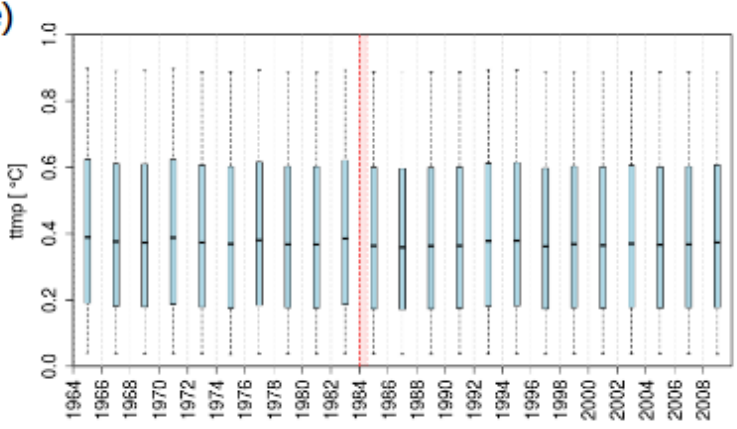

g)

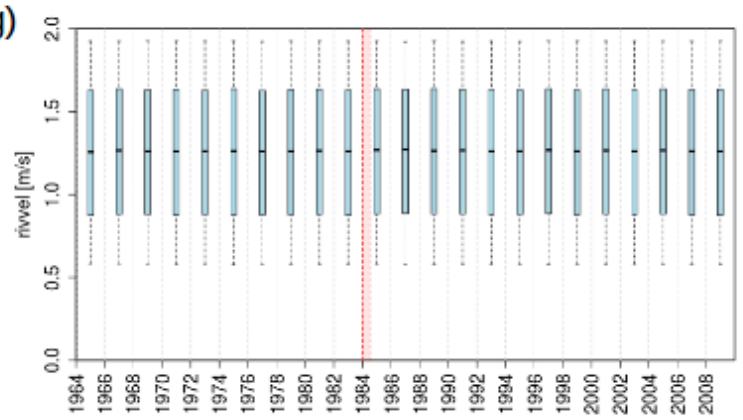

b)

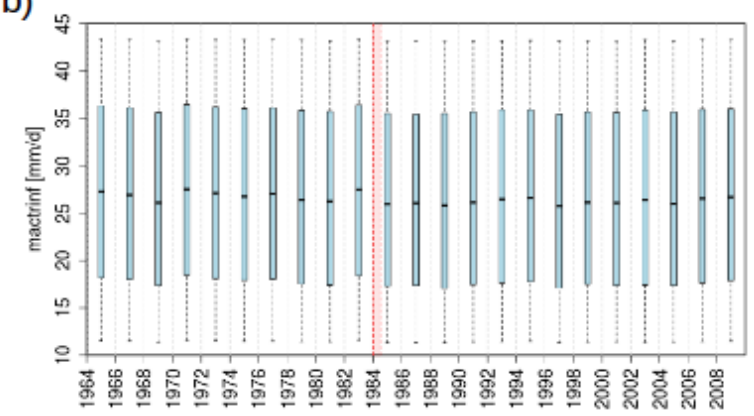

d)

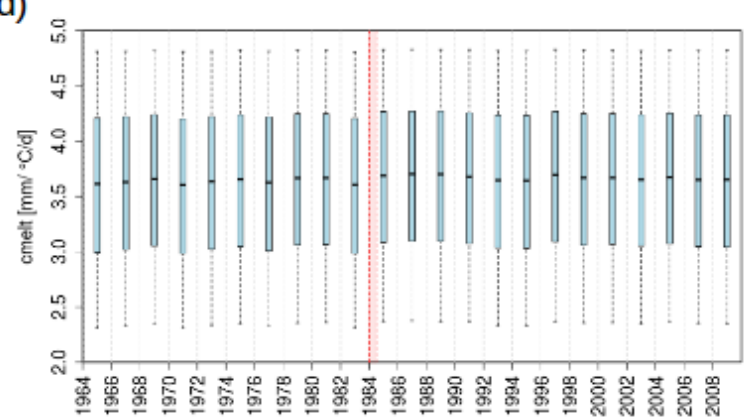

f)

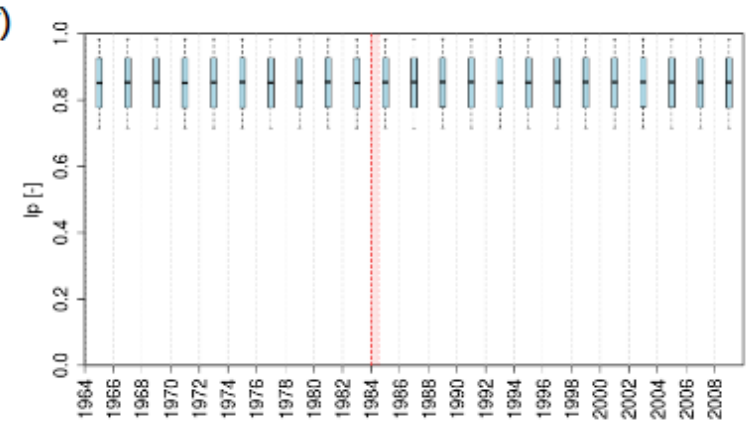

Figure S18. Continued posterior parameter distributions for the HYPE model in Hubbard Brook WS5. 


\subsection{HYMOD}

a)

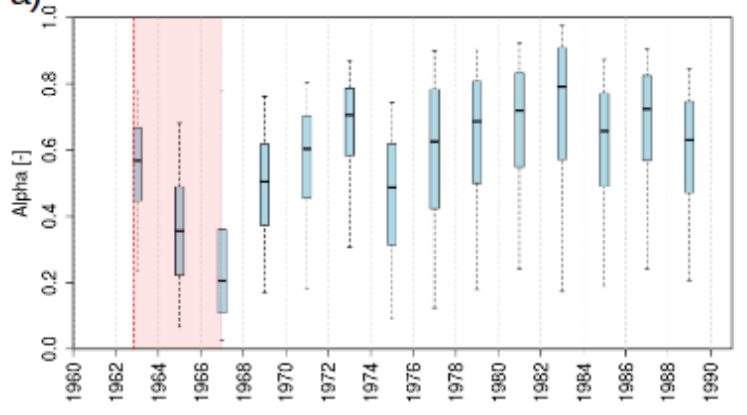

c)

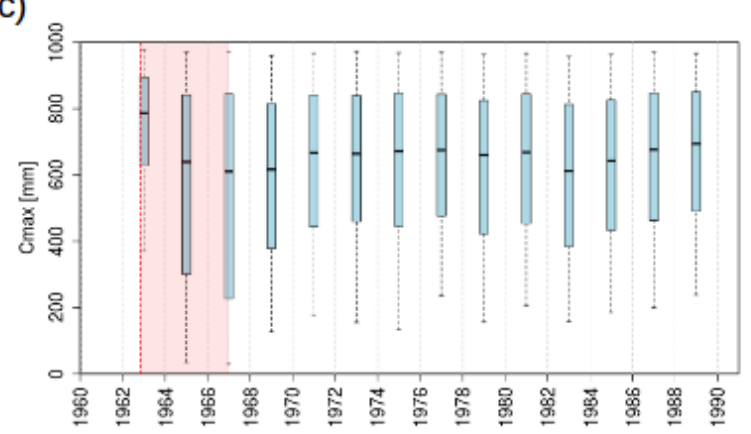

e)

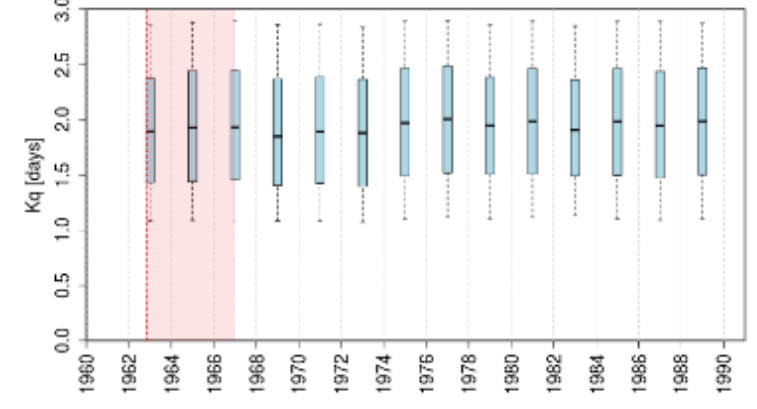

g)

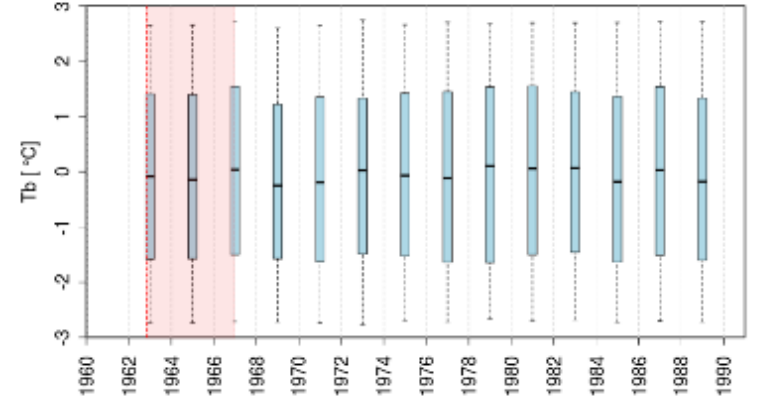

b)

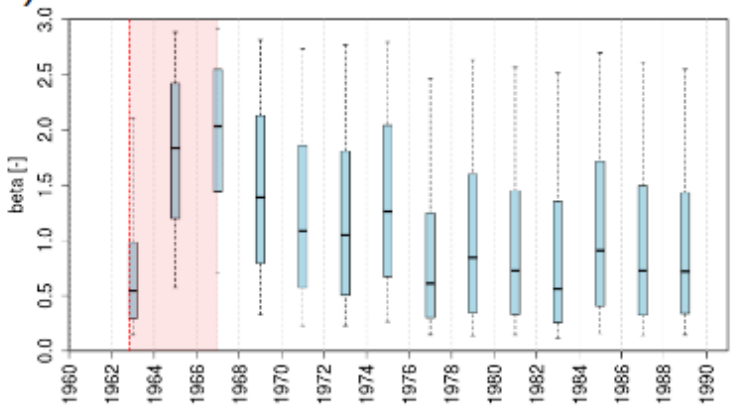

d)

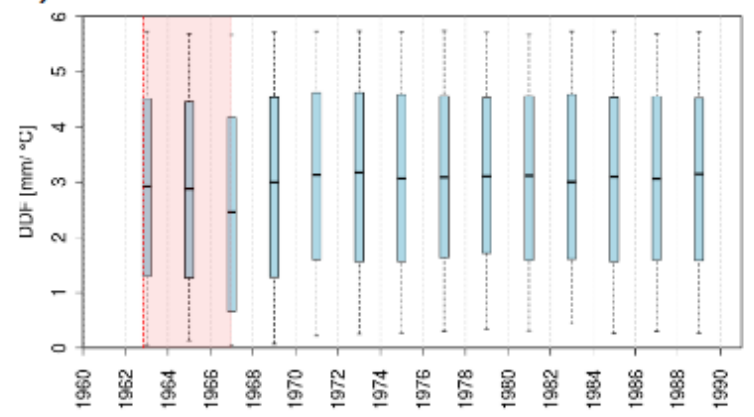

f)

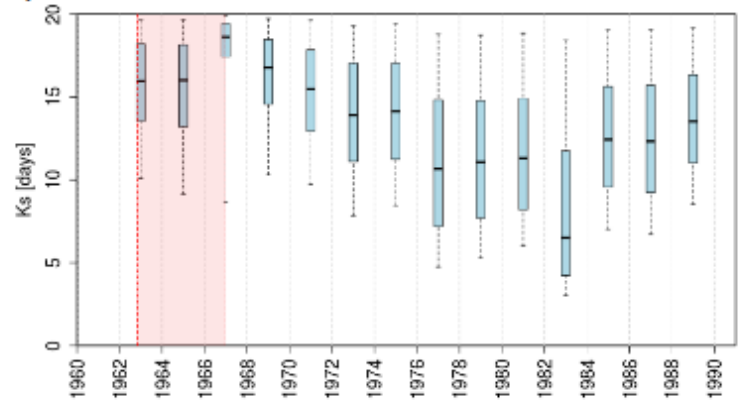

h)

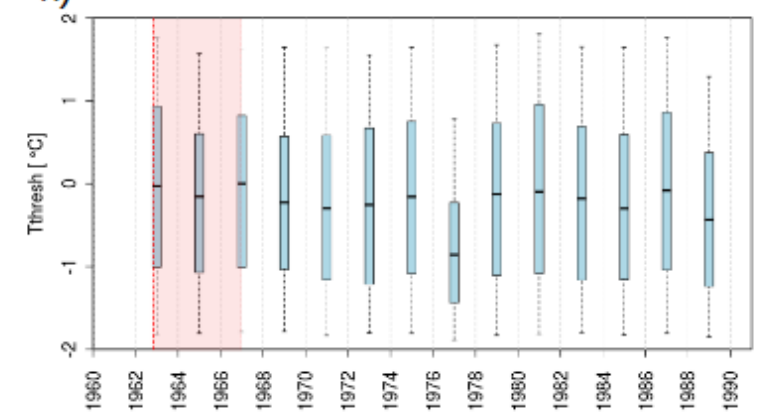

Figure S19. Posterior parameter distributions for the HYMOD model in HJ Andrews WS1. 
a)

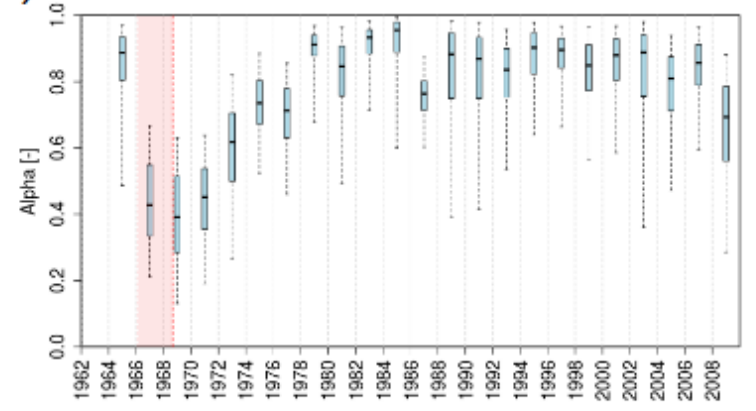

c)

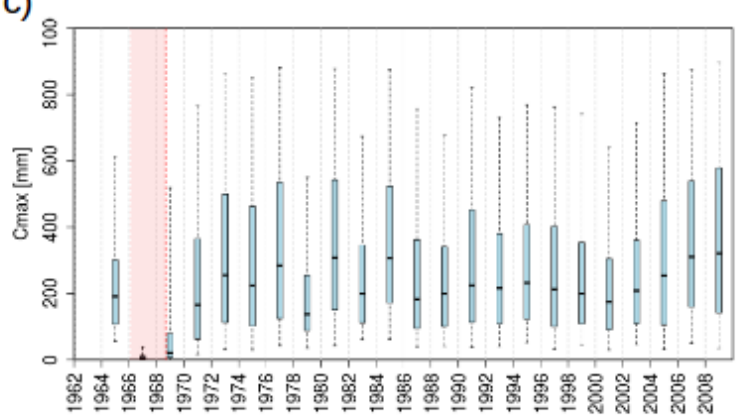

e)

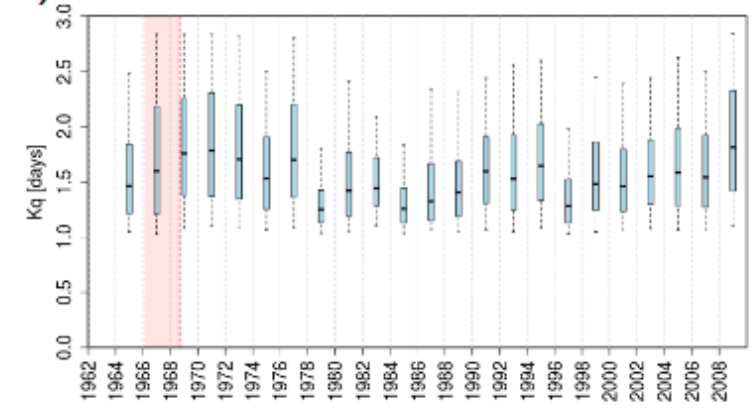

g)

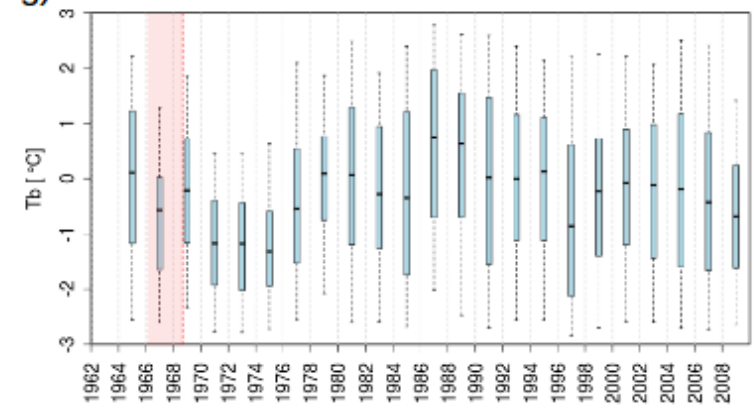

b)

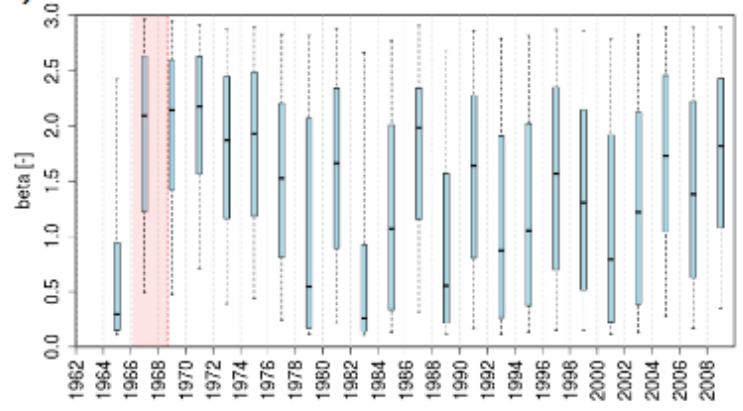

d)

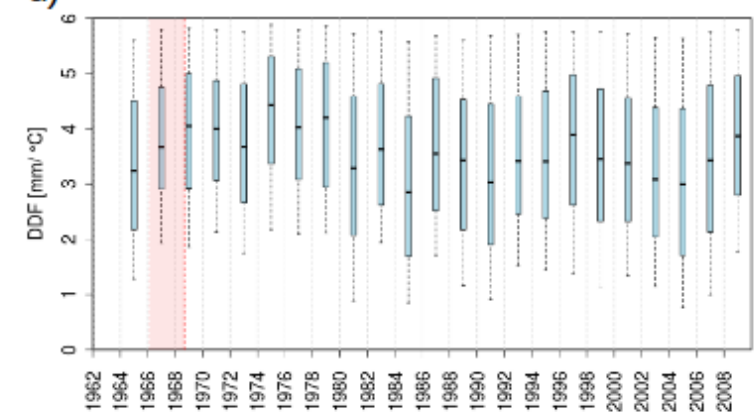

f)

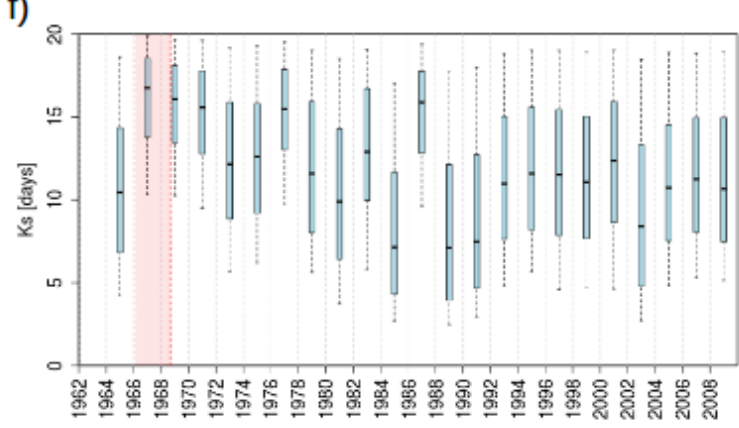

h)

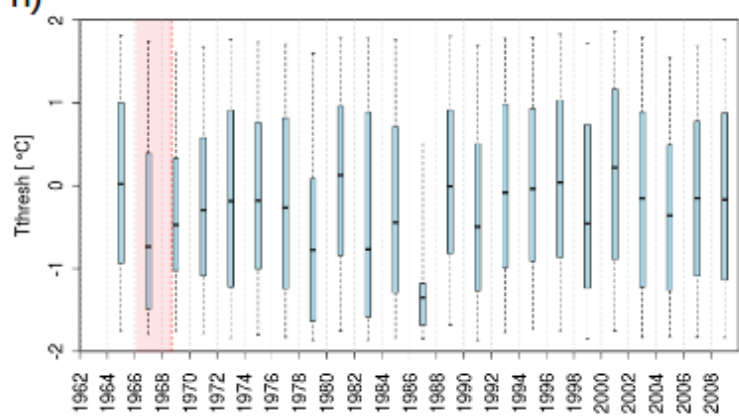

Figure S20. Posterior parameter distributions for the HYMOD model in Hubbard Brook WS2. 
a)

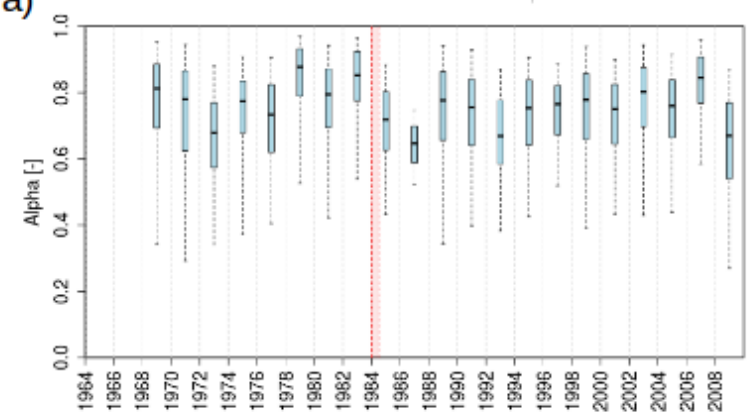

c)

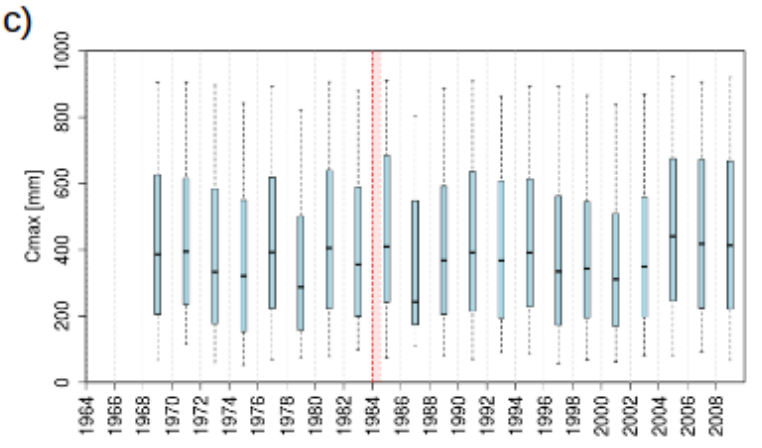

e)

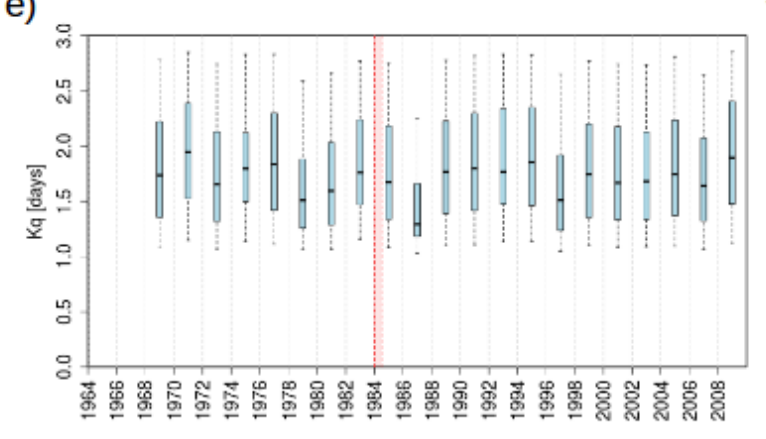

g)

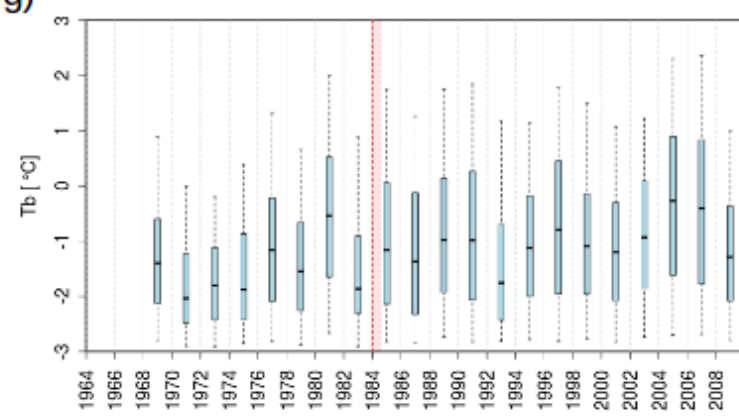

b)

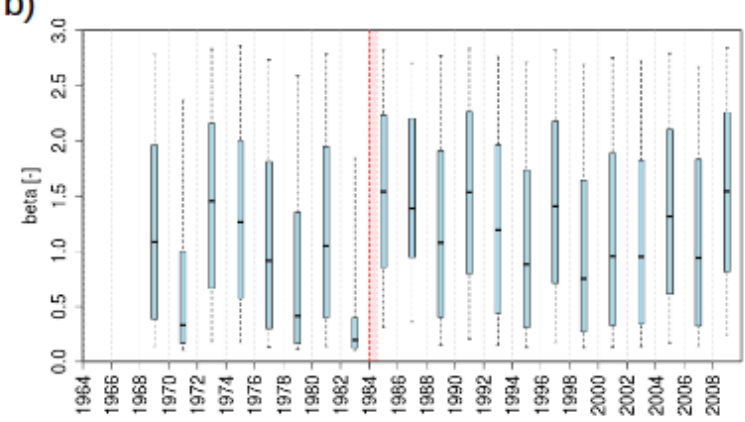

d)

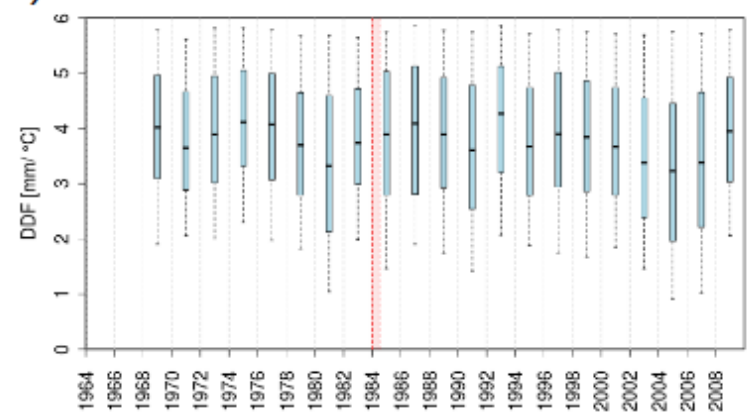

f)

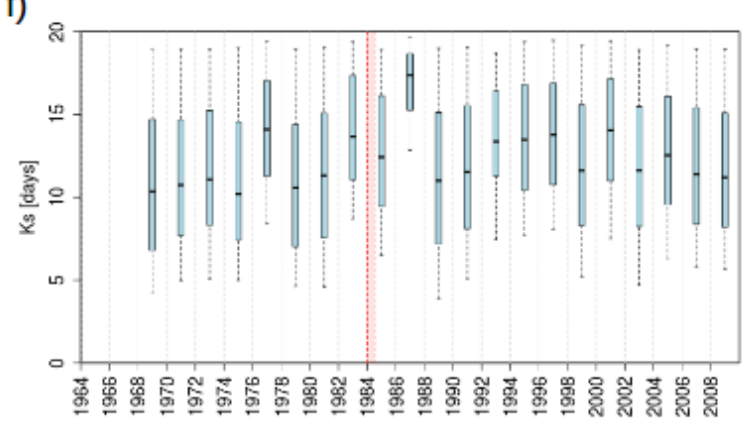

h)

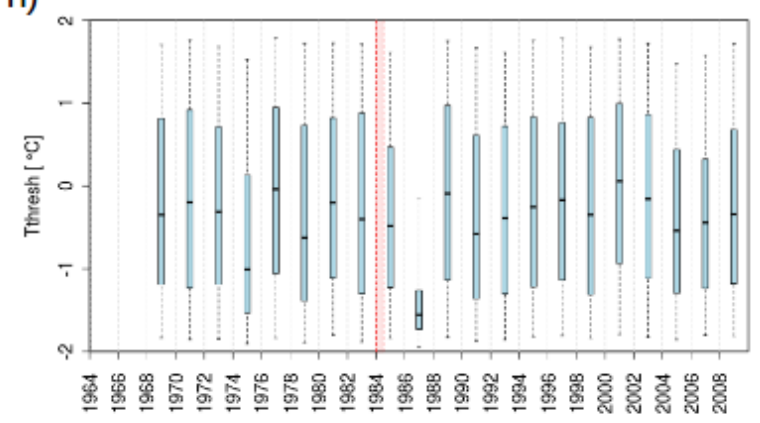

Figure S21. Posterior parameter distributions for the HYMOD model in Hubbard Brook WS5. 


\subsection{TUW}

a)

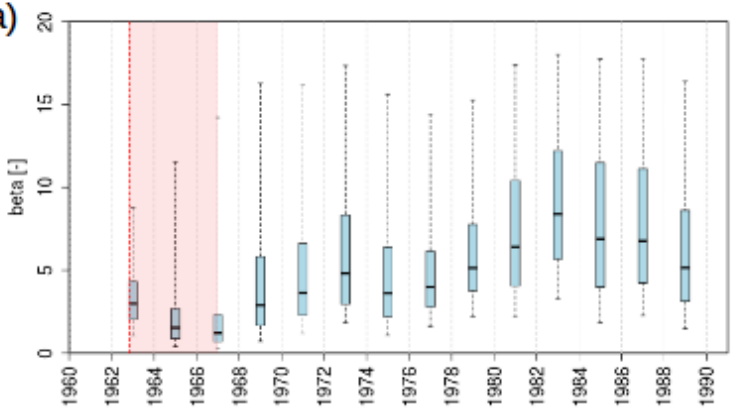

c)

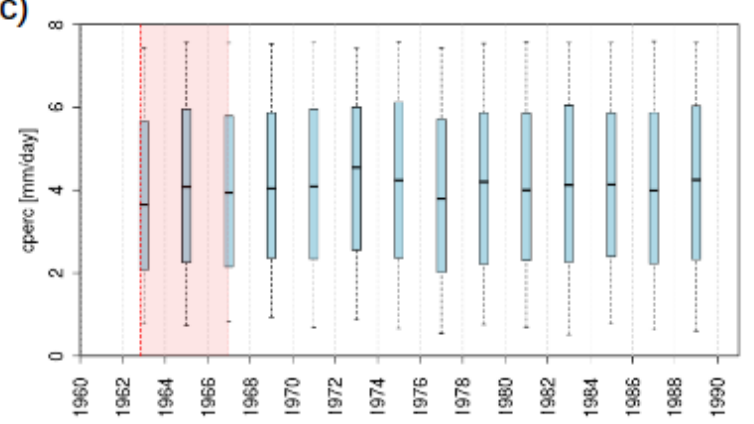

e)

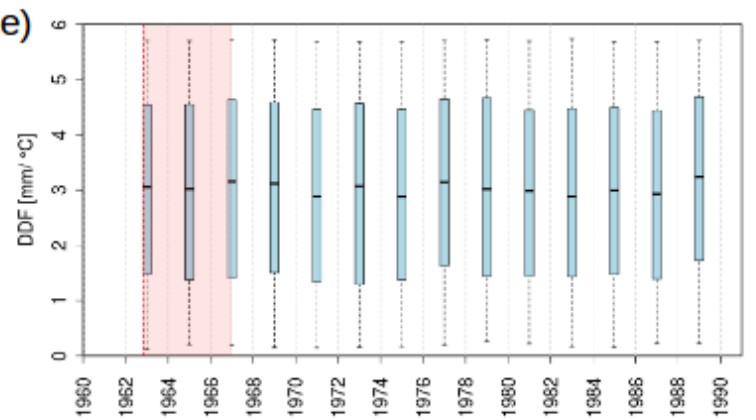

g)

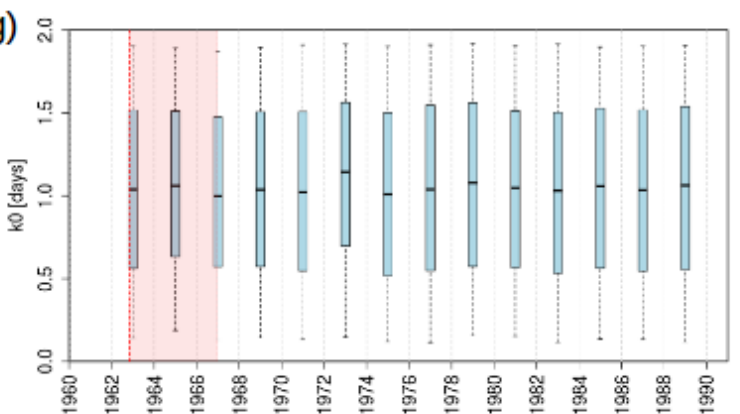

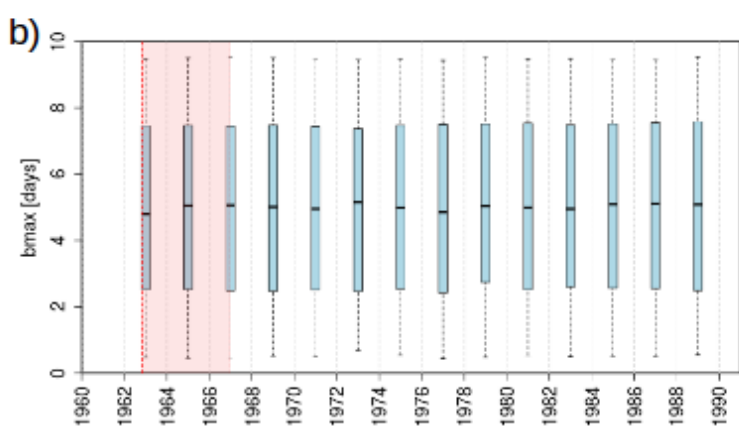

d)
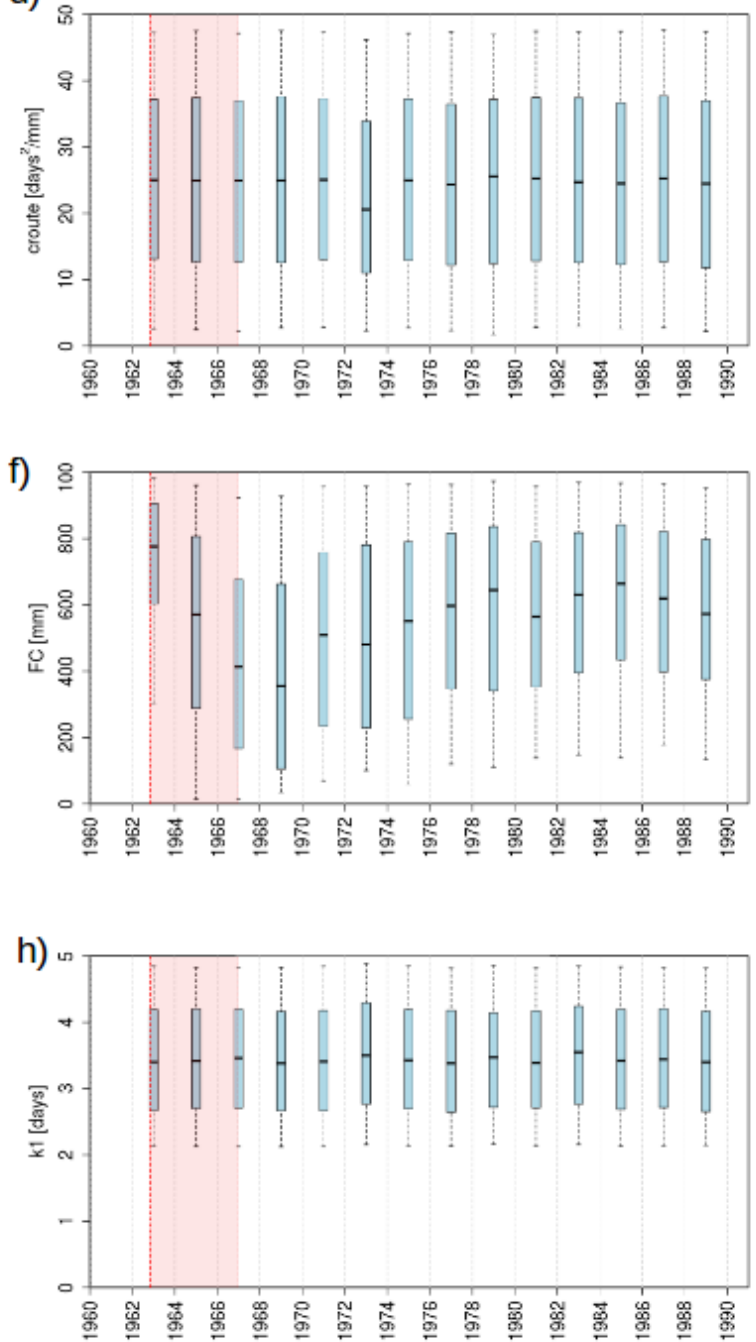

Figure S22. Posterior parameter distributions for the TUW model in HJ Andrews WS1. 
a)

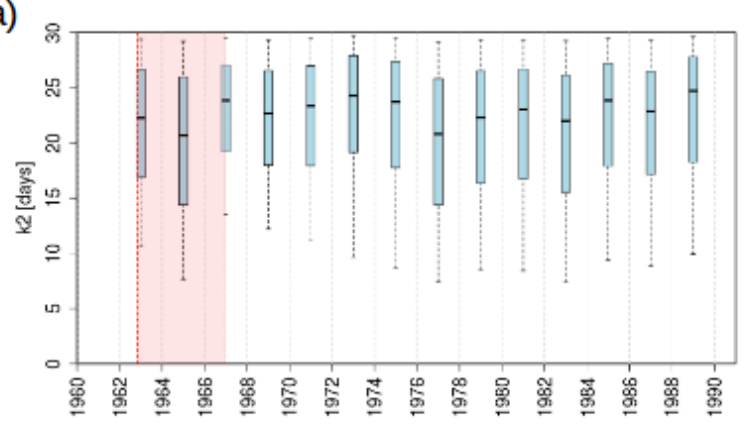

c)

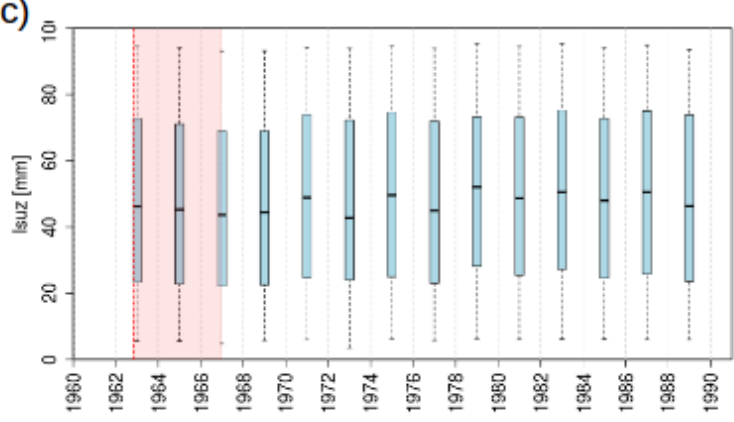

e)

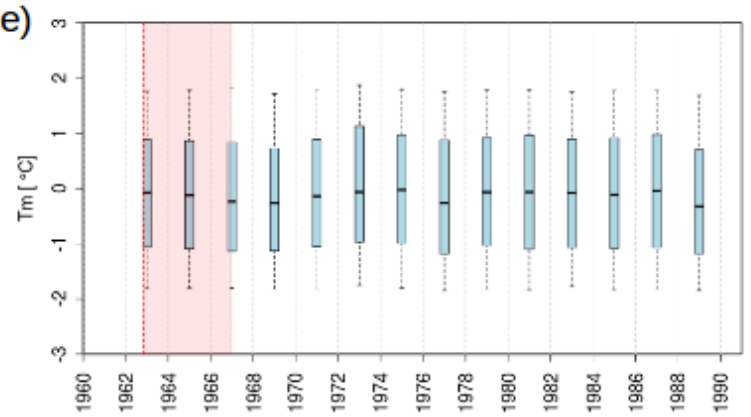

b)

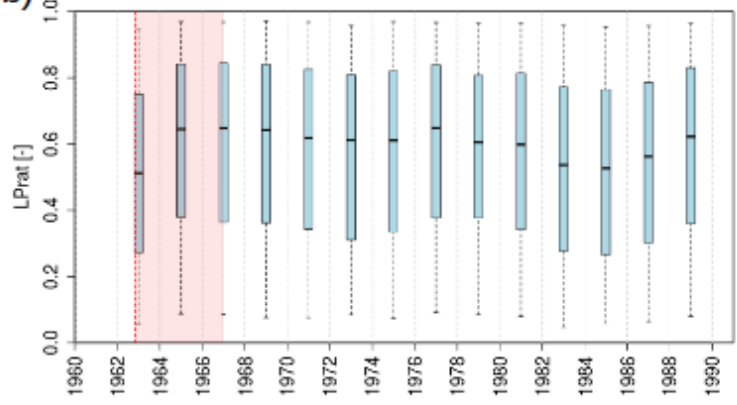

d)

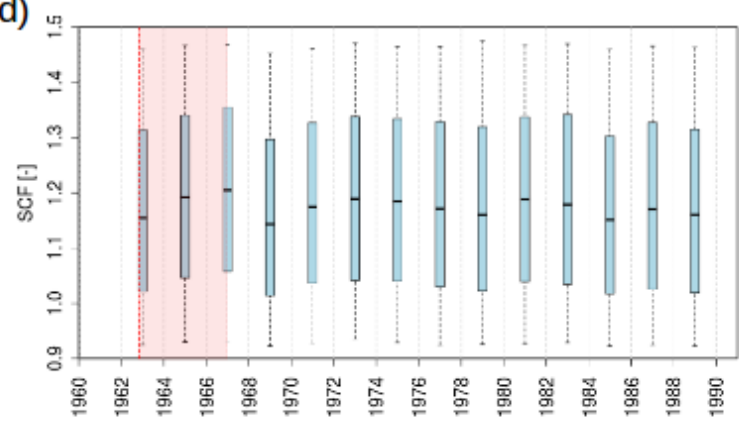

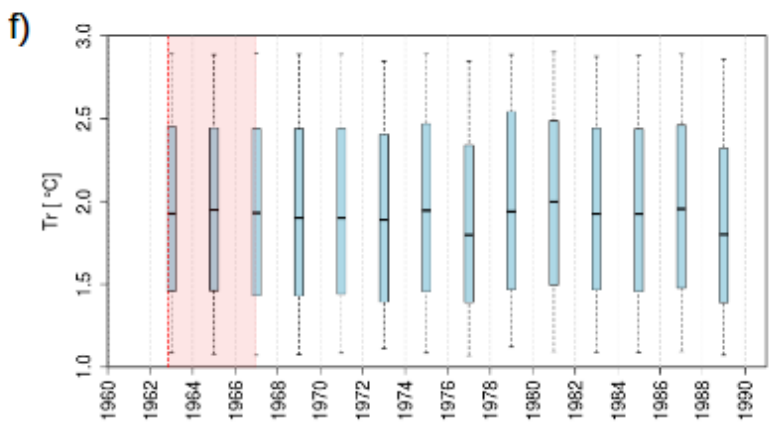

g)

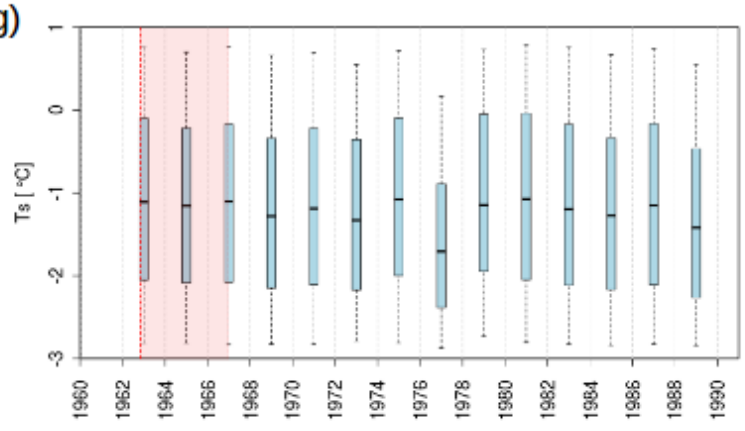

Figure S23. Continued posterior parameter distributions for the TUW model in HJ Andrews WS1. 
a)

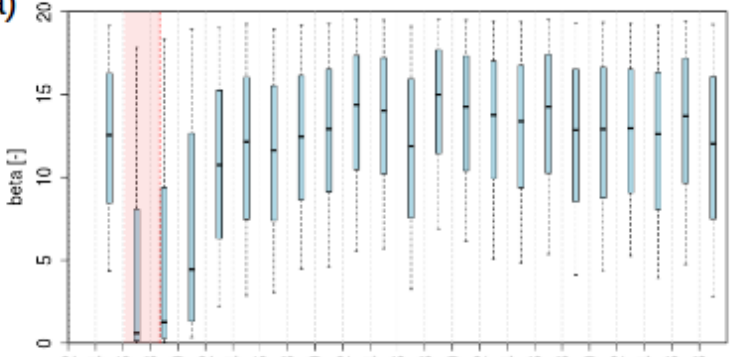

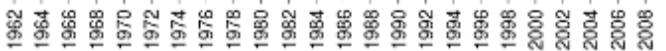

c)

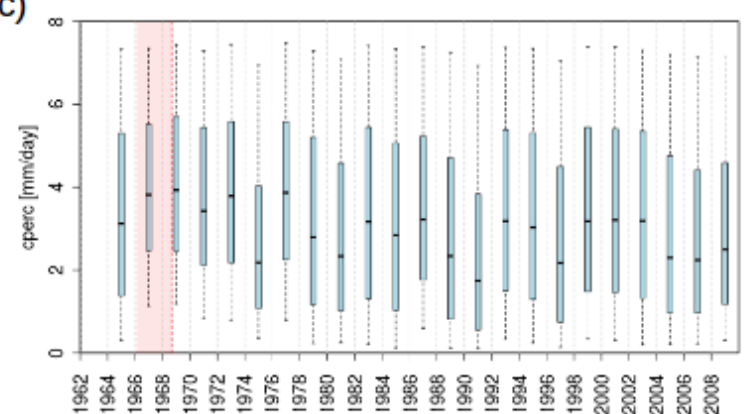

e)

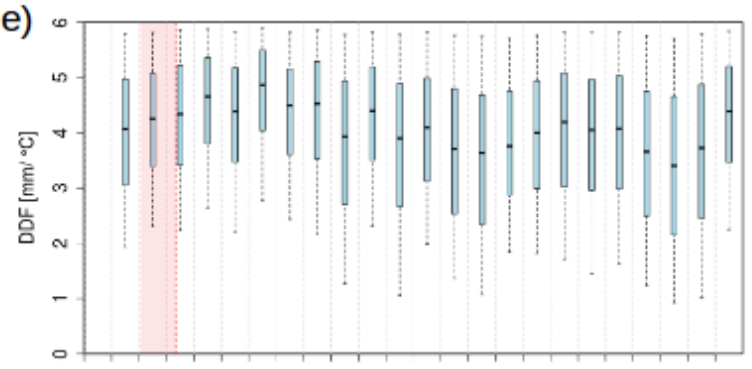

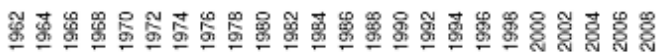

g)

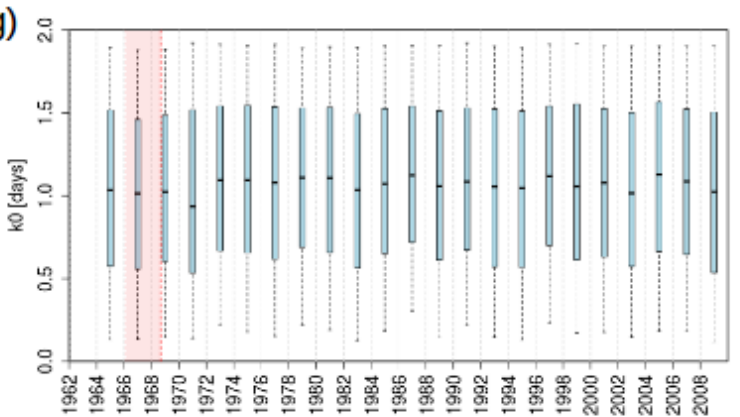

b)

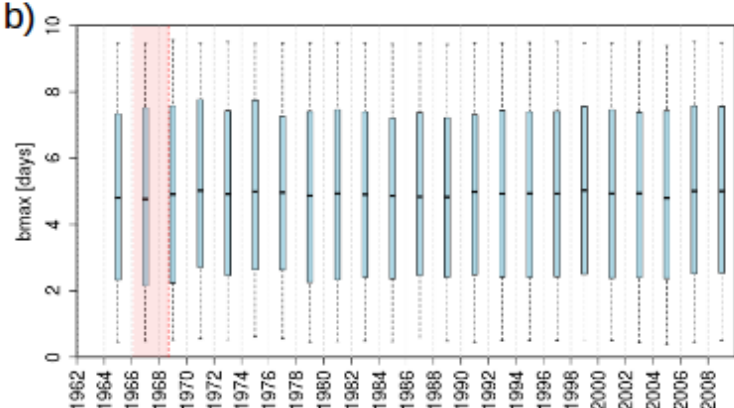

d)

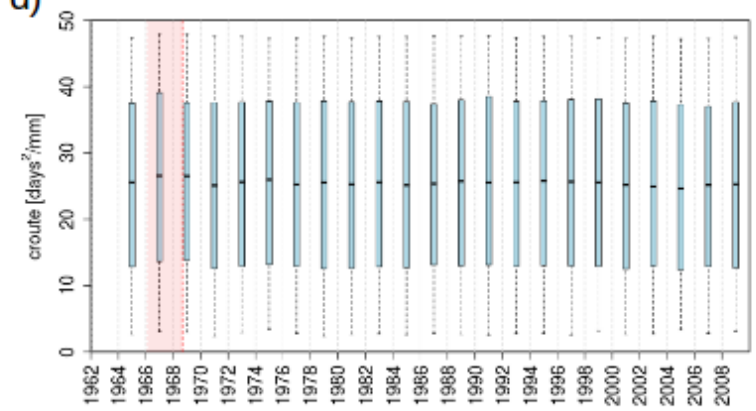

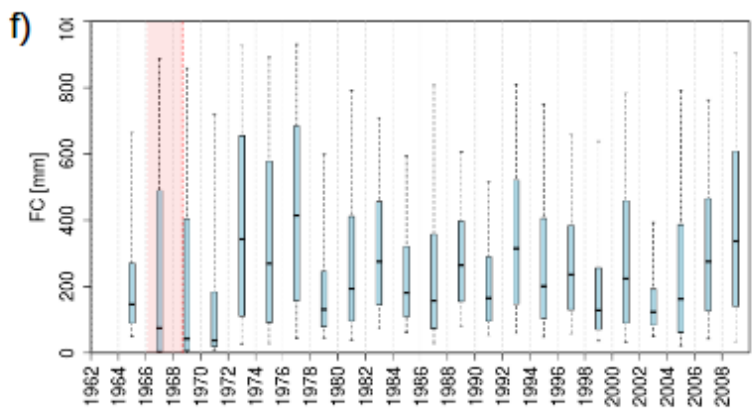

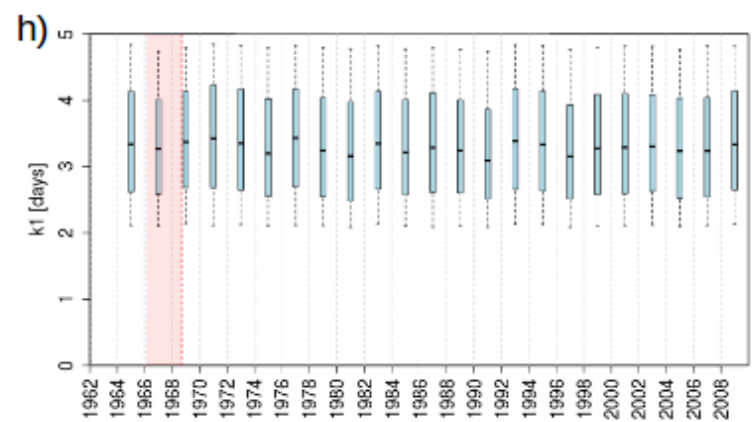

Figure S24. Posterior parameter distributions for the TUW model in Hubbard Brook WS2. 
a)
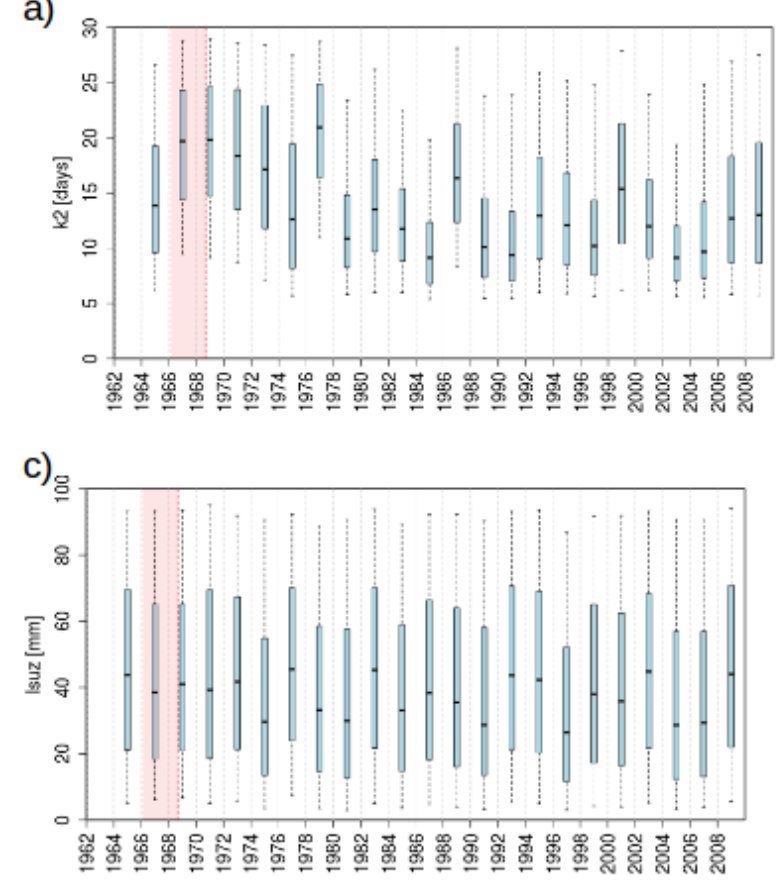

e)

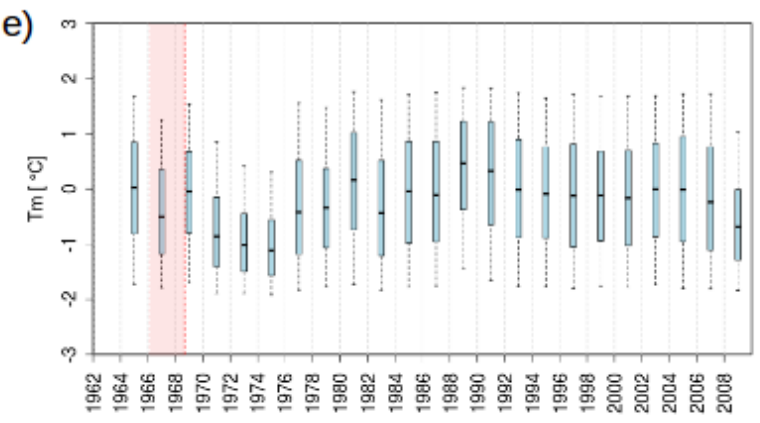

g)

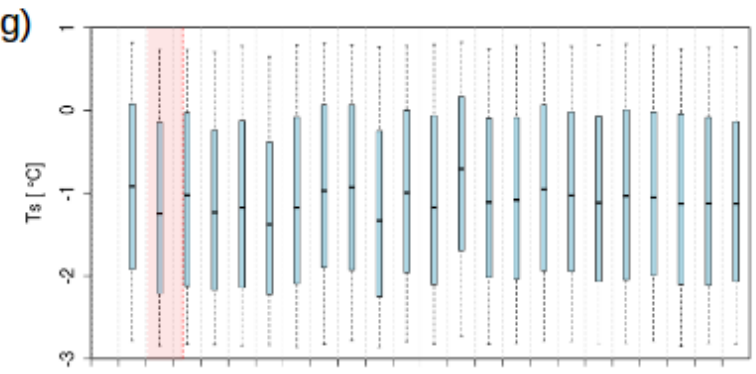

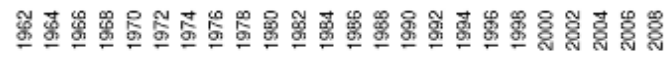
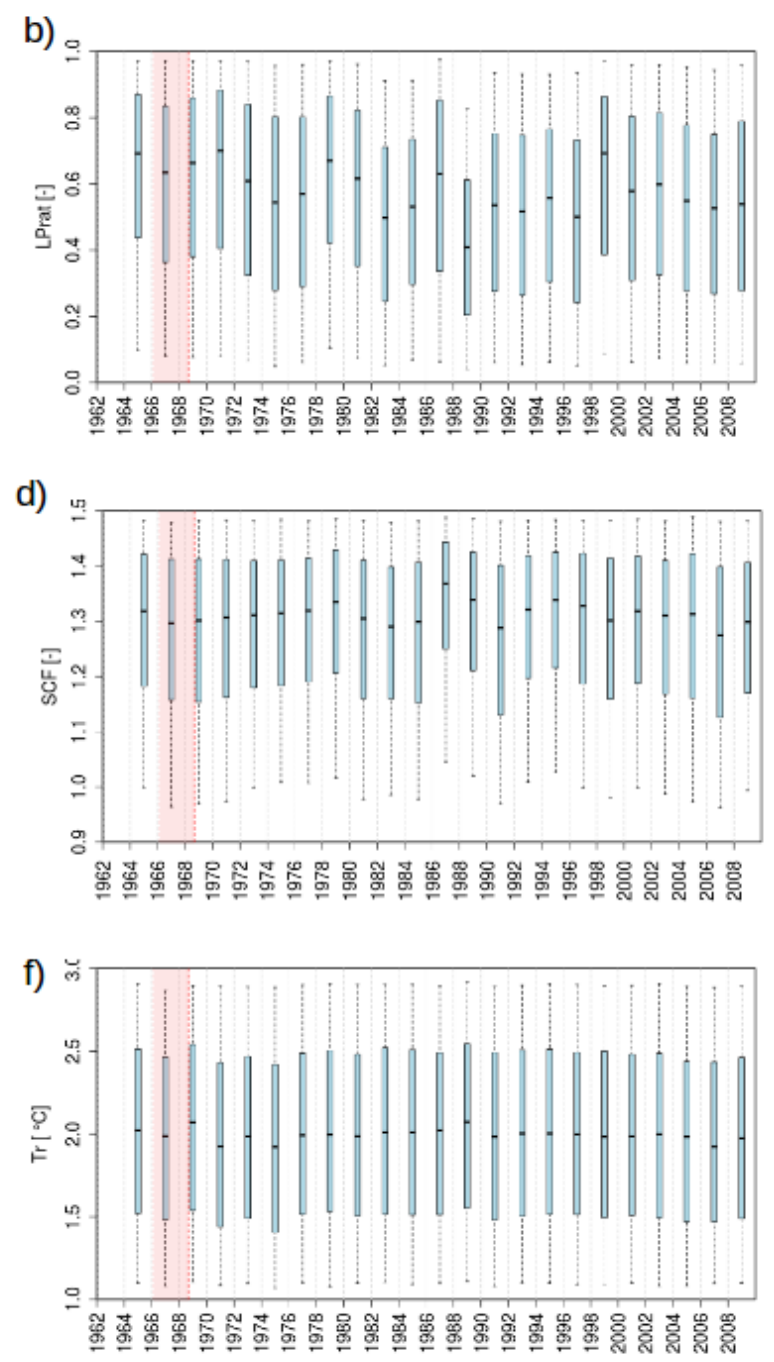

Figure S25. Continued posterior parameter distributions for the TUW model in Hubbard Brook WS2. 
a)

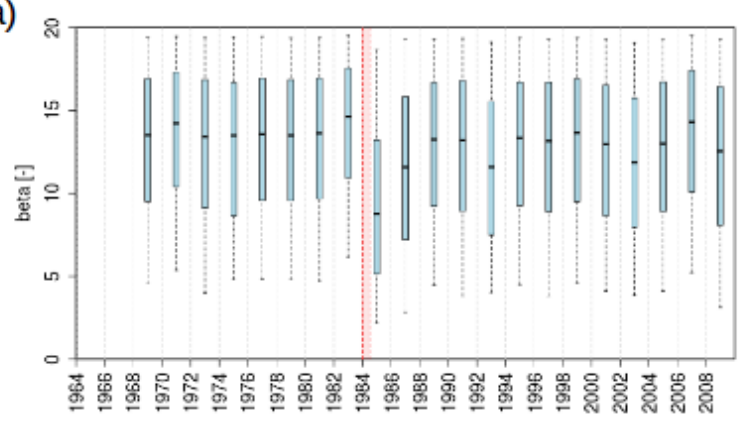

c)

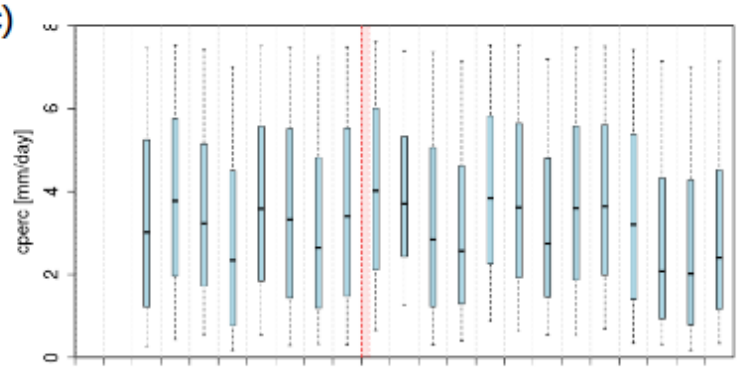

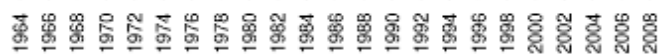

e)

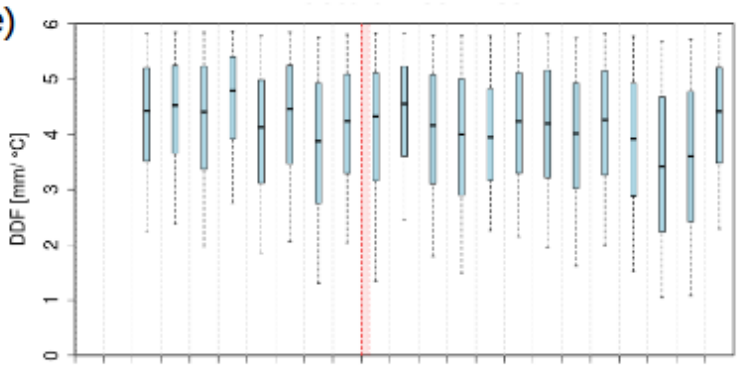

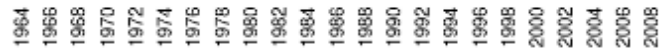

g)

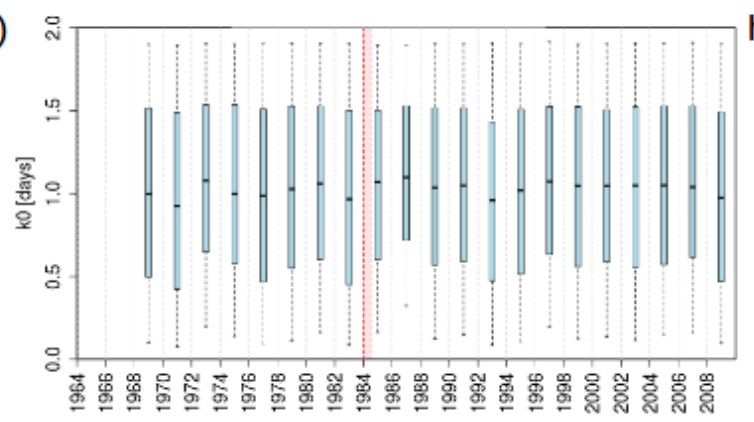

b)

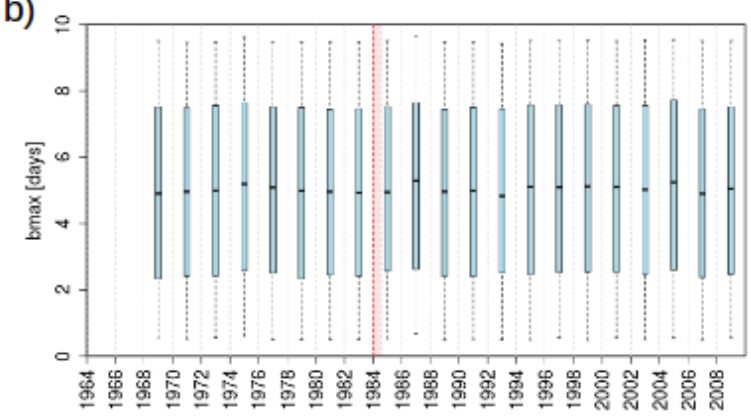

d)
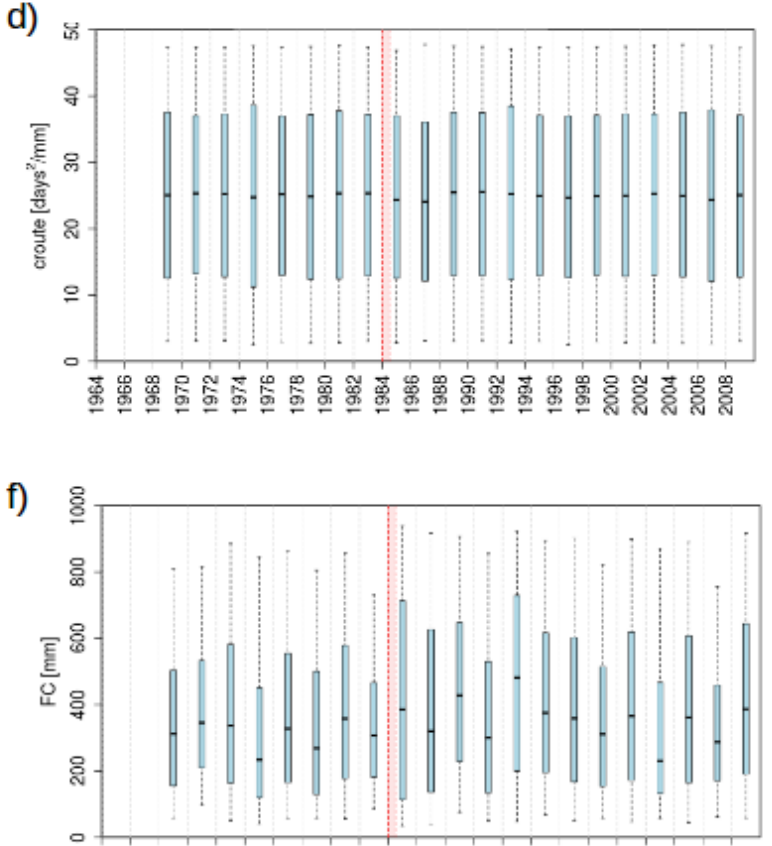

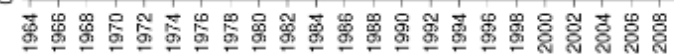

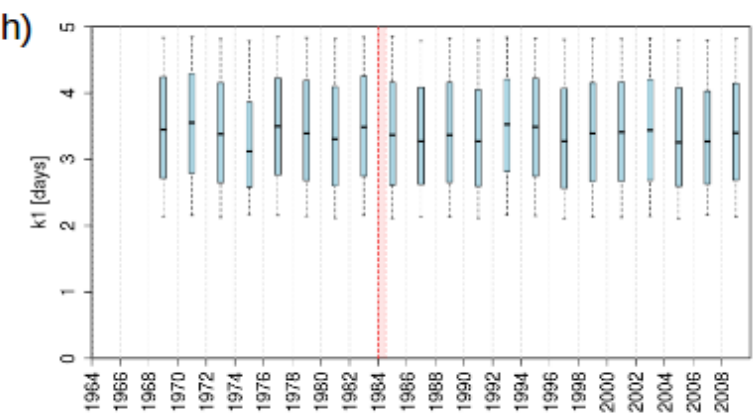

Figure S26. Posterior parameter distributions for the TUW model in Hubbard Brook WS5. 
a)

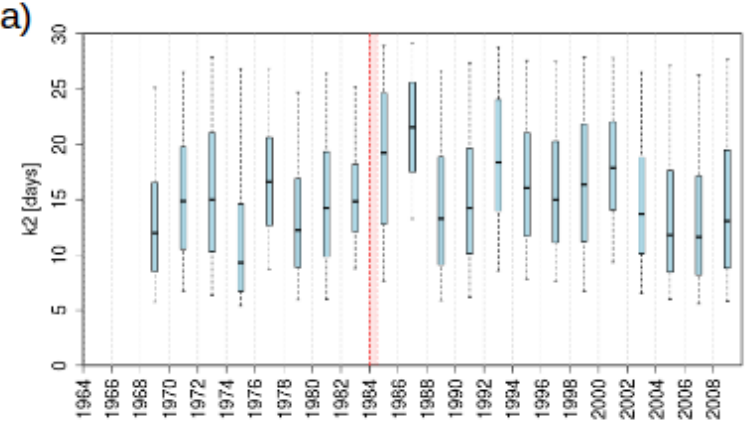

c)

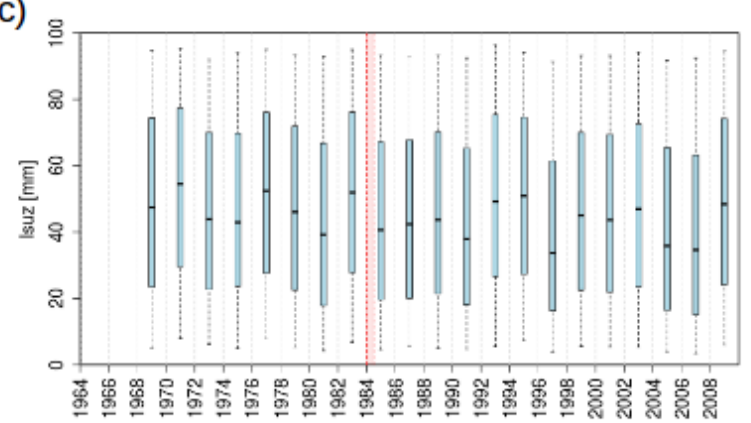

e)

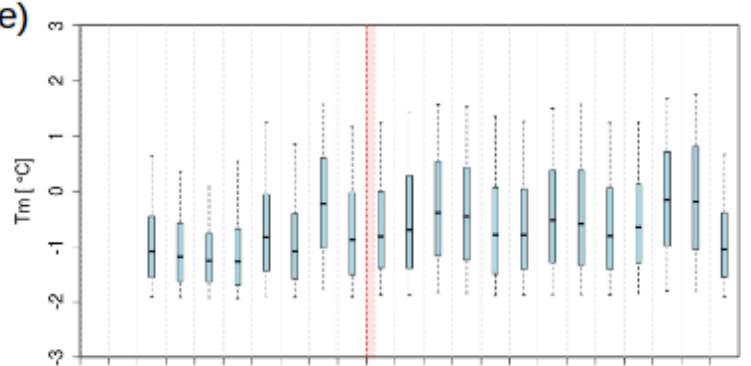

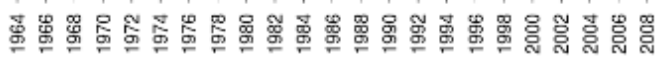

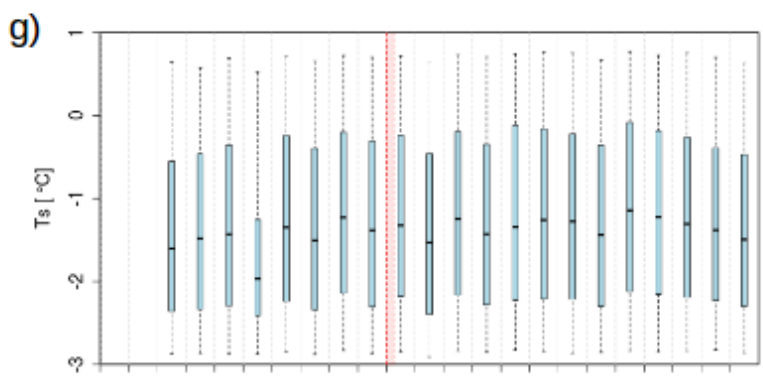

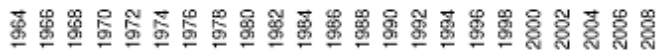

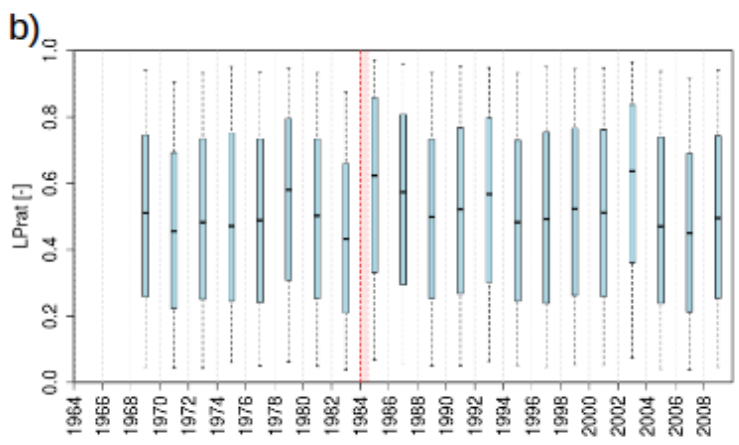

d)
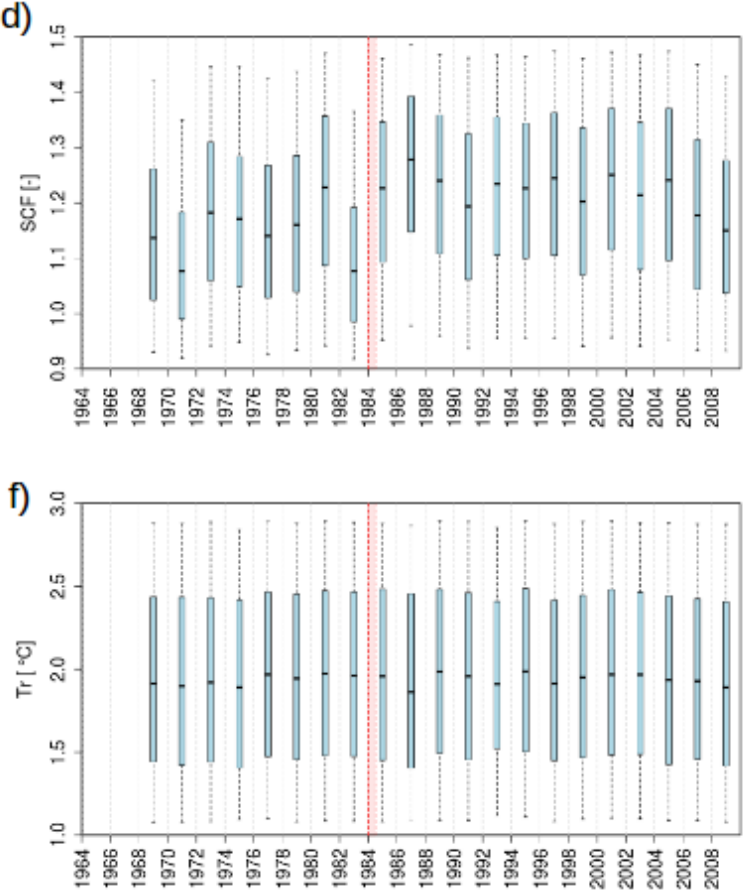

Figure S27. Continued posterior parameter distributions for the TUW model in Hubbard Brook WS5. 